\title{
Membrane permeability differentiation at the lipid divide
}

Urszula Łapińska ${ }^{1}$, Zehra Kahveci ${ }^{1, \$}$, Nicholas A. T. Irwin ${ }^{2,3}$, David S. Milner ${ }^{3}$, Alyson E. Santoro ${ }^{4}$, Thomas A. Richards $^{3^{*}}$, Stefano Pagliara ${ }^{*}$

\section{Affiliations:}

${ }^{1}$ Living Systems Institute and Biosciences, University of Exeter, Stocker Road, Exeter EX4 4QD, UK.

${ }^{2}$ Merton College, University of Oxford, Oxford OX1 4JD, UK

${ }^{3}$ Department of Zoology, University of Oxford, 11a Mansfield Road, Oxford OX1 3SZ, UK

${ }^{4}$ Department of Ecology, Evolution and Marine Biology, University of California, Santa Barbara, California 93106, USA

${ }^{\$}$ Current Address: Instituto de Nanociencia y Materiales de Aragón, CSIC-Universidad de Zaragoza, C/Pedro Cerbuna 12, Zaragoza 50009, Spain.

*Stefano Pagliara, Thomas Richards

Email: s.pagliara@exeter.ac.uk, thomas.richards@zoo.ox.ac.uk

https://orcid.org/0000-0003-3593-9248 (UL)

https://orcid.org/0000-0001-7931-8027 (ZK)

https://orcid.org/0000-0002-2904-8214 (NATI)

https://orcid.org/0000-0003-3669-7463 (DSM)

https://orcid.org/0000-0003-2503-8219 (AES)

https://orcid.org/0000-0002-9692-0973 (TAR)

https://orcid.org/0000-0001-9796-1956 (SP)

\section{Keywords}

Evolution, Vesicles, Microfluidics, Archaea, Bacteria, Prokaryote, Membrane transport, Transporters, Lipid bilayers 
bioRxiv preprint doi: https://doi.org/10.1101/2021.10.12.464042; this version posted October 13, 2021. The copyright holder for this preprint (which was not certified by peer review) is the author/funder, who has granted bioRxiv a license to display the preprint in perpetuity. It is made available under aCC-BY 4.0 International license.

\begin{abstract}
One of the deepest branches in the tree of life separates the Archaea from the Bacteria. These prokaryotic groups have distinct cellular systems including fundamentally different phospholipid membrane bilayers. This dichotomy has been termed the lipid divide and is assumed to bestow different biophysical and biochemical characteristics on each cell type. Classic experiments suggest that bacterial membranes are more permeable, yet systematic analysis based on direct measurements is absent. Here we develop a new approach for assessing the membrane permeability of cell-sized unilamellar vesicles, consisting of an aqueous medium enclosed by a single lipid bilayer. Comparing the permeability of twenty metabolites demonstrates that archaeal-type membranes are permeable to a range of compounds useful for core metabolic networks, including amino acids, sugars, and nucleobases. Surprisingly, permeability is much lower in bacterial-type membranes, in contradiction to current orthodoxy. We then show that archaeal permeability traits are specifically linked to both the methyl branches present on the archaeal phospholipid tails and the ether link between the tails and the head group. To explore this result further, we compare the abundance of transporterencoding families present on genomes sampled from across the prokaryotic tree of life. Interestingly, archaea have a reduced repertoire of transporter gene families, consistent with an increased dependency on membrane permeation for a subset of metabolites. Taken together, these results demonstrate that the lipid divide demarcates a clear difference in permeability function with implications for understanding some of the earliest transitions in cell and protocell evolution.
\end{abstract}




\section{Introduction}

Many models of the tree of life place the primary branch between the Bacteria (once called Eubacteria) and the Archaea (once called Archaebacteria) (1-5). Although this placement is debated (6-8), the Archaea/Bacteria bifurcation marks multiple important differences in cell biology, the mechanistic consequences of which are key to understanding the drivers of early cell evolution. Bacteria and Archaea are related, for example, possessing homologous ribosomal proteins (5) and RNA components (1), yet, Bacteria and Archaea possess distinct cell walls (10), DNA replication machineries (e.g. (11)), chromatin structures (12), and phospholipid membrane chemistries (13). This later characteristic, termed the lipid divide (14), is an important evolutionary factor considering: i) the hereditary potential of membranes; with most membranes generated by addition to the active in situ membrane and then passed from mother to daughter cell (15), and ii) the demonstration of viable prebiotic synthesis and self-assembly of membranes (for example through Fischer-Tropsch-type reactions (16)), and their catalytic and growth capabilities (for example in the presence of minerals and clays (17)).

Archaeal and bacterial phospholipid bilayers are assembled via distinct biosynthetic pathways (6). Most Bacteria possess membranes of fatty-acid chains linked to a glycerol-3-phosphate (G3P) backbone via ester bonds $(6,18)$ of right handed chirality $(13)$. In contrast, Archaeal membranes display a combination of diether or tetraether lipids (19) with isoprenoid chains containing methyl branches linked to a glycerol-1phosphate (G1P) backbone via ether bonds $(6,18)$ with left handed chirality $(13)$.

These differences mean that the spatial arrangements of phospholipids within the cell membrane are fundamentally different; such differences could have a profound impact on the primary function of cell membranes, which is to generate a semi-permeable solute barrier, allowing the development of chemical and proton gradients that drive the energetics and biochemistry of life (20). In vitro studies have identified important biological implications of the physico-chemical properties of phospholipid membranes by using liposomes (14, 21-29). Liposomes are approximately spherical synthetic lipid bilayer membranes with a typical diameter of $100 \mathrm{~nm}$ that enclose an internal aqueous phase. Using liposomes, a previous study suggested that prebiotically plausible membranes could acquire key metabolites from the environment in the absence of transport machinery such as transmembrane transporters (30). However, this work did not contrast the permeability of core metabolites across archaeal and bacterial-type membranes, but rather used mixtures of simple lipids, such as fatty acids, fatty alcohols and monoglycerides. An additional study has shown that, in the absence of counter-ions, diether archaeal lipid membranes display lower permeability to protons compared to bacterial lipid membranes (31). This feature was confirmed using independent approaches $(29,32)$ and is likely due to the difference in the ether vs ester tail-head link (33), and also constitutes an important trait for understanding differences in energetic functions of the Archaea and Bacteria (20). It was also shown that bacterial and diether archaeal lipid membranes display similar permeabilities to glycerol, urea and ammonia (31). However, these permeability traits were measured at high extracellular metabolite concentration (i.e. $200 \mathrm{mM}$ ) using indirect spectroscopic techniques, which average over a large number of liposomes and associated impurities (e.g. multi-lamellar liposomes and lipid aggregates). As such there are a number of potential limitations of these results: i) they are based on ensemble measurements across variant lipid architectural forms, ii) they do not investigate permeability characteristics of cellular metabolites at biologically/ecologically realistic substrate concentrations, iii) they investigate a limited number of metabolites, and iv) liposomes used in these studies had smaller dimensions and greater membrane curvature compared to many living cells, where curvature is known to play an important role in molecular membrane interactions $(34,35)$. Collectively, this means that we 
have a limited understanding of the implications of membrane chemistry variation on metabolite permeability in biologically relevant contexts, particularly given recent evidence suggesting that living cells can obtain nutrients from the environment in a transporter-independent fashion (36).

Here we test the hypothesis that different membrane types have different permeability traits. Such a difference would mean that cells, or protocells (i.e. simple primitive cells made of replicators surrounded by lipids (37)), with alternative membrane chemistries could access different metabolites independent of membrane transporter proteins. It has been proposed that early cell evolution involved the association of a self-replicating hereditary molecule (perhaps RNA) within a phospholipid membrane (38). This idea has been termed the 'Obcell Hypothesis' and involves the eventual incorporation of a self-replicating hereditary molecule within a phospholipid bilayer. For such a system to function, metabolites, which are necessary for a self replicator, need to cross the membrane either by permeation or by the evolution of a transport system, such as transmembrane proteins. If bacterial and archaeal lipid bilayers possessed differing permeability traits then it would mean that the ancient Archaea/Bacteria bifurcation would also encompass a distinct change in metabolite permeability. Such a difference would have profound effects for the evolution of membrane transporter repertoires, intracellular metabolic networks, and associated cellular ecologies.

We present a new approach for the study of membrane permeability based on microfluidic manipulation of unilamellar vesicles composed of a single phospholipid bilayer of archaeal or bacterial membrane mimics. Collectively, we show that the lipid divide demarcates a dichotomy in membrane permeability characteristics, with permeability traits the opposite of what has previously been assumed because archaeal membranes display elevated permeability to many key compounds. Using phylogenomic approaches, we also demonstrate that this functional difference correlates with key variations in the evolution of the transporter protein encoding gene repertoire.

\section{Results and discussion}

Microfluidic screening to explore membrane permeability characteristics

Using microfluidics, we developed a system to enable the capture and individual placement of unilamellar vesicles approximating cellular sizes in the range of 5 to $15 \mu \mathrm{m}$ in multiple parallel arrays of tens of vesicles (Figure 1). Although these are on the larger end of prokaryotic cell sizes, these dimensions were chosen to aid imaging and manipulation while minimising curvature effects (34). This approach enabled us to precisely control the chemostatic fluid environment of the vesicles. In order to measure metabolite permeation into the unilamellar vesicles, we loaded the vesicles with carboxyfluorescein (CF), a fluorescent dye which allows direct assessment of molecular uptake by variations in fluorescent properties in response to changes in intra-vesicle metabolite concentration $(31,39)$. An increase in intra-vesicle fluorescence indicates membrane permeability to the target metabolite when delivered via continuous flow through the microfluidic device (Figure 1 and Methods). Individual metabolites were delivered into the extra-vesicle environment, while imaging the diffusion of these molecules across the membrane of multiple individually trapped vesicles (Methods, Figure 1 and Video S1). By combining these technologies, we conducted parallel controlled experiments exploring how cellular metabolites can cross membranes of different phospholipid chemical composition.

Employing this approach, we studied the permeability of single vesicles composed of eight different lipid chemistries representing simplified archaeal and bacterial membranes (see Table S1) to twenty small metabolites (Table S2). First, we tested seven amino acids with different molecular weights, hydrophobicities, charges, and numbers of rotatable bonds. Strikingly, the archaeal membrane mimic (lipid 1 in Table S1) was 
consistently more permeable to five amino acids compared to the bacterial membrane mimic (lipid 2 in Table $\mathrm{S} 1$, Figure $2 \mathrm{~A}-2 \mathrm{E}$ ), the only exceptions being tryptophan and lysine. While tryptophan, an aromatic amino acid with the highest molecular weight, was impermeable across both membrane types (Figure 2F), both membranes were similarly permeable to lysine, the only positively charged amino acid tested (Figure S1). However, for both the archaeal and bacterial membrane mimics, we did not find a significant correlation between permeability and amino acid molecular weight, hydrophobicity, or rotatable bond number (Pearson coefficient $r=-0.55,-0.56,-0.16$, respectively, for the archaeal membrane mimic; $r=0.40,0.34,0.57$, respectively, for the bacterial membrane mimic; none significant at $p=0.05$; Table $S 2$ ). These data suggest that the metabolic selectivity of these membrane mimics is complex and does not rely only on basic molecular properties such as molecular weight or hydrophobicity. The archaeal membrane mimic was most permeable to glycine (Figure 2A), the smallest amino acid investigated. Archaeal membrane permeabilities to alanine and glutamine were heterogeneous (Figure 2B and 2E, coefficient of variations -CV- of $213 \%$ and $103 \%$ ) in accordance with the notion that lipid membranes are a system with heterogeneous functions $(40,41)$. It is also worth noting the different dynamics in the uptake of the different amino acids, with alanine displaying the steeper uptake at short time-scales followed by a plateau (Figure $2 \mathrm{~B}$ ), whereas glutamine uptake did not reach plateau levels within the experimental time-frame (Figure 2E).

Next, we tested six uncharged sugars with different molecular weights, hydrophobicity, and number of rotatable bonds (see Table S2). Consistent with the amino acid findings, the archaeal membrane mimic was more permeable to a subset of these metabolites compared to the bacterial membrane mimic (Figure 3). However, the difference in permeability was reduced between the archaeal membrane compared to the bacterial membrane for relatively small sugars such as glyceraldehyde and dihydroxyacetone (Figure $3 \mathrm{~A}$ and 3B, respectively).

The difference in permeability was strongly distinct for two larger sugar types, deoxyribose and ribose; primary constituents of DNA and RNA, respectively (Figure 3D and 3E). Again, we did not find a significant correlation between the permeability of a sugar and its molecular weight, hydrophobicity or the number of rotatable bonds present (Pearson coefficient $r=0.62,-0.60,0.06$, respectively, for the archaeal membrane mimic; $r=0.26,-0.15,-0.66$, respectively, for the bacterial membrane mimic; none significant at $p=0.05$; Table S2), corroborating previous findings obtained using simple fatty acid membranes (39). Therefore, additional second-order properties of sugar compounds must be considered. These data corroborate the hypothesis that subtle variations in the metabolite atomic structure can lead to large differences in membrane permeability ( 39 , 40, 42).

Finally, the archaeal membrane mimic was more permeable to the amide urea, the pyrimidine uracil, the phosphonate 2-aminoethyl phosphonic acid, and the purines, adenine and guanine (Figure 4A, 4C, 4D, $4 \mathrm{E}$ and $4 \mathrm{~F}$ respectively), compared to the bacterial membrane mimic. Overall, we did not find a significant correlation between metabolite permeability and their molecular weight or hydrophobicity or number of rotatable bonds (Pearson coefficient $r=0.09,-0.20,0.03$, respectively, for the archaeal membrane mimic; $r=0.32,0.09,-0.03$, respectively, for the bacterial membrane mimic; none significant at $p=0.05$; Table S2). We also attempted to measure permeabilities to the nucleotide adenosine monophosphate, however, vesicles of both membrane mimics burst during its delivery.

Importantly, these differences in permeability traits were not due to vesicle deformation during metabolite uptake, since the dimensions of each vesicle did not significantly change during metabolite delivery (Figure S2, slope non-significantly different from zero for all metabolites and membrane mimics investigated). 
Such differences were also not attributable to differential metabolite accumulation within the lipid bilayers, since: i) CF has very low affinity for the lipid hydrophobic chains (31) so interactions with substrates within the membrane are unlikely, and ii) CF fluorescence intensity was uniform across whole vesicles for both the membrane mimics and the full range of metabolites investigated, consistent with previous data obtained on fatty acid and phospholipid liposomes $(31,39)$. Taken together these data suggest that the archaeal membrane mimic displays a range of elevated permeability traits to small metabolites compared to the bacterial membrane mimic. These differences are not due to leakages through the archaeal membrane mimic as our data also demonstrate that the archaeal membrane mimic is selectively permeable to only a subset of the metabolites. Specifically, the archaeal membrane mimic is impermeable to tryptophan in a similar fashion to the bacterial membrane mimic, while it is also impermeable to glyceraldehyde, despite the latter compound being half the size. Furthermore, the uptake of each metabolite across the archaeal membrane mimic is characterised by unique dynamics (see for comparison Figure $2 \mathrm{~B}$ and $2 \mathrm{E}$ ), demonstrating that metabolites are not passing across this membrane via puncture holes generated in the membrane during vesicle formation but by genuine diffusion through the lipid bilayer in a metabolite-specific manner. Finally, we wanted to rule out that the relatively lower permeability of the bacterial lipid mimic could be ascribed to interactions between different lipids within the ternary lipid mixture that we employed to mimic more closely bacterial membranes (lipid 2 in Table S1). To do so, we measured and contrasted permeability to urea, glycine, ribose, deoxyribose, glycerol and phosphonate in bacterial ternary-lipid mixtures (lipid 2 in Table S1) and bacterial membranes made of a single lipid (lipid 3 in Table S1) and found that these two different bacterial mimics displayed comparably low permeabilities to all the metabolites tested (Figure S3).

\section{Which archaeal lipid characteristics determine permeability traits?}

Next, to uncover the chemical determinants of archaeal membrane permeability, we investigated the impact of variation in lipid chemistry on permeability traits of the archaeal membrane mimic. First, we tested the impact of the lipid chain branching and tail-head link, two hallmarks of archaeal lipids, on the permeability of urea, glycine, ribose, deoxyribose, glycerol and phosphonate. We found that in the absence of lipid chain branching, the archaeal membrane mimic (lipid 4 in Table S1 and black diamonds in Figure 5) displayed a statistically significant and consistently lower permeability compared to the archaeal membrane mimic with lipid chain branching (lipid 1 in Table S1 and green circles in Figure 5). This striking difference is possibly due to increased membrane fluidity in the presence of branched lipid chains $(30,43)$. In the absence of branching, the archaeal membrane mimic displayed permeabilities to urea, glycine, deoxyribose or ribose that were comparable to the permeabilities measured for the bacterial membrane mimic (lipid 2 in Table $S 1$ and blue squares in Figure 5A, $5 B, 5 E$ and $5 F$, respectively, none significantly different at $p=0.05$ ); whereas permeabilities to glycerol and phosphonate were lower in the archaeal membrane mimic without branching compared to the bacterial membrane mimic (Figure $5 \mathrm{C}$ and $5 \mathrm{D},{ }^{* * *}$ and ${ }^{* *}$, respectively).

In the absence of ether link (substituted with an ester link) the archaeal membrane mimic (lipid 5 in Table S1 and magenta triangles in Figure 5) displayed significantly lower permeabilities compared to the archaeal membrane mimic with the ether link (lipid 1 in Table S1 and green circles in Figure 5), and exhibited permeabilities similar to the bacterial membrane mimic (lipid 2 in Table $S 1$ and blue squares in Figure 5, none significantly different). Taken together these data strongly suggest that in order to achieve elevated permeability traits, lipids must simultaneously feature an ether link and methyl chain branching, both of which characterize archaeal lipids. 
To follow on from this, we then set out to determine whether permeability traits are affected by variations in the archaeal lipid head (lipid 6 in Table S1). However, despite attempting different electroformation protocols (see Table S3) we could not produce vesicles using this lipid, possibly because this lipid forms nonlamellar structures of either a cubic or hexagonal fashion (44).

Next, we investigated how permeability variability varies in archaeal phospholipids according to chain length. These experiments provide no evidence of a significant correlation between lipid chain length $(\mathrm{C}=12$, $C=16$ and $C=18$, i.e. lipids 7,4 and 8 in Table $S 1$ ) and the permeability to any of the metabolites investigated (Figure S4, Pearson correlation coefficient $r=-0.58,0.95,0.52,-0.10,-0.69$ and 0.30 for urea, glycine, glycerol, phosphonate, deoxyribose and ribose, respectively), corroborating previous findings on eukaryotic membrane mimics (45). A short chain length slightly favoured permeability to urea and deoxyribose (Figure S4A and S4E, respectively), whereas a long chain length slightly favoured permeability to glycine and ribose (Figure S4B and $\mathrm{S} 4 \mathrm{~F}$, respectively), but overall, these effects were masked by vesicle-to-vesicle variation in permeability to these metabolites. Furthermore, we attempted to produce vesicles using lipids of other chain lengths $(\mathrm{C}=6$ and $C=14$, i.e. lipids 9 and 10 in Table S1); however, these vesicles appeared to be mechanically unstable, possibly because their transition temperature is close to the temperature at which we carried out our membrane permeability assays.

We next demonstrated that bonding saturation (i.e. single bonds that were more likely present in prebiotic molecules (30)) along archaeal phospholipids of fixed chain length ( $C=18$, lipid 8 in Table S1) significantly decreased permeability to the small amide, urea, and, to a lesser extent, to the small amino acid, glycine, compared to bonding unsaturation (i.e. double bonds, lipid 11 in Table S1, Figure S5A and S5B, *** and ${ }^{*}$, respectively). However, bonding saturation did not have an impact on the permeability to glycerol, phosphonate, deoxyribose, or ribose (Figure S5C, S5D, S5E and S5F, respectively, none significantly different), whereas a previous study using liposomes found increased permeability to ribose in unsaturated fatty acids (30).

Aromatic rings along the lipid chains or tetraether links could further affect the permeability traits in archaeal membrane mimics. Given the variance of these lipid forms across Archaea (19) and the observation that such variants are often the minority constituents of archaeal membranes $(46,47)$ we suggest they do not directly reflect the core archaeal membrane form. However, we do acknowledge that introduction of these lipid variations is likely to alter permeability traits. The experimental platform presented is readily adaptable to investigate the effect of further chemistry variations, however, these are not currently commercially available. One alternative is using lipids isolated and purified from cell culture. However, such an approach could introduce membranes bearing transmembrane proteins, fragments of transmembrane proteins and/or variant lipid 'rafts' limiting our ability to control for chemical and structural heterogeneity of the lipid mixtures which compose cell membranes. Future work should explore the effect of lipid mixtures on permeability traits. Furthermore, we did not investigate the effect on permeability of membrane variants embedding an S-layer, a peptidoglycan layer, or an outer membrane such as those seen in diderm bacteria, because all these features are predicted to follow the evolution of a protocell and in some cases must also have followed the archaeal/bacterial bifurcation. We also acknowledge that permeability traits can vary with changes in environmental conditions such as $\mathrm{pH}$, temperature or salinity levels $(48,49)$ and the differences we have observed are likely subject to variation in different environmental conditions. Although our experimental platform could be adapted to investigate permeability traits under variant environmental conditions, there are constraints as vesicles suffer damage in the presence of very low or high $\mathrm{pH}$ and/or salinity. Moreover, lipids 
change state above or below the transition temperature. Therefore, the experimental platform would need further development to physically stabilise vesicles, by using, for example, higher density media or by forming vesicles on physical support structures.

\section{Membrane permeability negatively correlates with transporter gene repertoires}

The observed differences in membrane permeability implies that any transition between archaeal and bacterial-type membrane chemistries would require extensive recalibration of numerous cellular systems in response to changes in permeability, osmotic stress, and metabolite homeostasis. Such a transition could be facilitated by a hybrid or mixed membrane $(6,50)$, but the ultimate change would require adaptation to these altered cellular properties, for example, the evolution of membrane transporters could permit a reduction in lipid membrane permeability. Accordingly, given the increased permeability of archaeal lipid membranes, we hypothesized that archaeal genomes would encode a significantly reduced complement of transporter gene families relative to bacteria, particularly for those protein families known to transport metabolite types capable of permeating archaeal lipid membranes (shown in Figures 2, 3 and 4). A limited transporter repertoire could reflect a reduced dependency on protein-based translocation systems as metabolite requirements could be satisfied by a combination of core metabolic function and lipid membrane diffusion.

To test this hypothesis, we iteratively searched diverse bacterial $(n=3,044)$ and archaeal $(n=243)$ genome-derived predicted proteomes using profile hidden Markov models (HMM, $n=277$ ) derived from TCDB (Transporter Classification Database) protein families (51), to identify previously classified transporter homologs across prokaryotes. Despite the sensitivity of our search, the Archaea displayed a reduced transporter repertoire relative to the Bacteria, irrespective of bacterial membrane system (e.g., monoderms or diderms) (Figure 6A). Certain transporter families were consistently encoded across prokaryotes, including many ion transporters (see cluster 2 in Figure 6A, largely composed of ion transporters), which are required due to the impermeability of membranes to ions, with the exception of protons (45). In contrast, other families showed reduced representation in Archaea (see clusters 1, 3, and 5 in Figure $6 \mathrm{~A}$ which were functionally heterogeneous, whereas cluster 4 comprised outer membrane transporters associated with diderms). In particular, transporter families known to translocate metabolites similar to those that permeate archaeal membrane mimics (e.g., amino acids, sugars and nucleobases, Figures 2, 3 and 4, respectively) were significantly depleted, independent of taxon sampling (Figure 6B).

It has previously been argued that protein-membrane interactions, attuned to the specific lipid characteristics of either bacterial or archaeal membranes, may have acted to enforce the lipid divide as the Bacteria and Archaea diversified (14). Therefore, to account for the possibility that archaeal transporters were not accurately recovered in our searches due to divergent biochemical characteristics or a lack of archaeal transporter family representation in TCDB, we first examined the possibility that archaeal membrane transporters have transmembrane (TM) domains with different lengths or alternative amino acid compositions, a factor that may have obscured previous HMM based annotations of transporter gene families. Comparisons between over 10.8 million bacterial and 528,000 archaeal TM domains (identified from over 2.2 million bacterial and 116,000 archaeal transporter proteins) revealed no significant differences in TM domain characteristics between Archaea and Bacteria (Figure S6), suggesting that these biochemical properties would neither impair bioinformatic detection nor would limit horizontal gene transfer of transporter proteins from Bacteria to Archaea (14). Secondly, to identify putative archaeal transporters that may have been absent from the TCDB database, we clustered archaeal proteins into protein families and identified those with characteristics indicative of 
transporter proteins (a median of at least four TM domains and annotation with transporter-associated PFAM functional domains). This search revealed only thirteen previously unsampled putative transporter families (mostly branched-chain amino acid permeases, ABC- and EamA-transporters) that were predicted to function in metabolite uptake and were present in at least a quarter of archaeal species (Supplementary file 1). However, only a single undetected putative $A B C$-transporter family was identified in over $50 \%$ of archaeal species $(70.8 \%)$. These results strongly indicate that unidentified transporters do not account for the reduced transporter repertoire observed in Archaea (Supplementary file 1). Together, these results are consistent with a reduced requirement for certain metabolite transporter proteins in Archaea, an evolutionary outcome that has likely been driven by the increased metabolite permeability of archaeal lipid membranes which reduces the requirement for protein transporters and also must limit their utility.

\section{Conclusion}

Close to the origins of cellular life, each cell-like assembly likely possessed a variant membrane composition, representing a diversity of lipid heads, head-tail linkages, tail lengths, saturation, and branching. Protocell assemblies displaying a combination of lipid features favourable for proto-metabolic function would have had a selective advantage; allowing a protocell enclosing sustainable metabolic function to emerge and proliferate. Here we demonstrate that archaeal and bacterial lipid membranes show distinct differences in permeability characteristics, with archaeal-type lipid membranes showing permeability to a range of compounds that would theoretically be useful to a cellular or protocellular metabolic network. Indeed, our data demonstrate that archaeal membrane permeability is dependent on methyl chain branching and ether linkage properties, two hallmarks of archaeal lipids. Such discriminatory permeability functions would provide a protocell with a compartment containing only a selection of specific metabolic resources, which would define the pool from which a proto-metabolic network could arise. Taken together with previous findings, demonstrating that archaeal lipid membranes are impermeable to ions, these data invoke the suggestion that a protocell without a dedicated transmembrane transportation system would be more likely to acquire stable and diversified metabolic functions using an archaeal lipid membrane than a bacterial lipid membrane. We therefore suggest that the presence of transport machinery was not a prerequisite for the emergence of a protocell bearing an archaeal lipid membrane chemistry. As such, the acquisition of features such as transport machinery during early cellular evolution could follow, for example, the transition from simple fatty acid membranes (39) to archaeal phospholipid membranes. Furthermore, archaeal selectivity towards ribose and deoxyribose over other sugars might have allowed faster assimilation of these sugars; hardwiring the use of these substrates for hereditary materials and the fixation and successive emergence of RNA and DNA as hereditary encoders of biological information. Together with compelling data demonstrating that isoprenoids enhance the stability of lipid vesicles that self-assemble on minerals (25) and encapsulate primordial nucleic acids absorbed on their surface (21), our findings suggest that a prebiotic archaea-like lipid membrane could accommodate a range of heterotrophic functions and that a heterotrophic origin of primitive cellular life from an archaeal lipid world is therefore more feasible than an alternative scenario based on a bacterial phospholipid membrane. 


\section{Materials and methods}

\section{Preparation of materials}

All chemicals were purchased from Merck. All lipids [i.e. lipid 1 (1,2-di-O-phytanyl-sn-glycero-3phosphocholine), lipid 2 (1,2-dioleoyl-sn-glycero-3-phosphoethanolamine, 1,2-dioleoyl-sn-glycero-3-phospho(1'-rac-glycerol), 1,3-bis (sn-3'-phosphatidyl)-sn-glycerol), lipid 3 (1,2-dioleoyl-sn-glycero-3phosphoethanolamine), lipid 4 (1,2-di-O-hexadecyl-sn-glycero-3-phosphocholine), lipid 5 (1,2-diphytanoyl-snglycero-3-phosphocholine), lipid 6 (1,2-di-O-phytanyl-sn-glycero-3-phosphoethanolamine), lipid 7 (1,2-di-Ododecyl-sn-glycero-3-phosphocholine), lipid 8 (1,2-di-O-octadecyl-sn-glycero-3-phosphocholine), lipid 9 (1,2di-O-hexyl-sn-glycero-3-phosphocholine), lipid 10 (1,2-di-O-tetradecyl-sn-glycero-3-phosphocholine) and lipid 11 (1,2-di-O- (9Z-octadecenyl)-sn-glycero-3-phosphocholine), see Table S1] were purchased from Avanti Polar Lipids within Merck. Indium tin oxide (ITO) coated glass slides were purchased from VisionTek Systems Ltd. The fluorescent probe 5(6)-carboxyfluorescein (CF), (MW=376 g/mol), was dissolved in absolute ethanol at a stock concentration of $10 \mathrm{mg} / \mathrm{mL}$. In order to perform all permeability experiments at physiological $\mathrm{pH}$ (7.4), the washing buffer was prepared by dissolving sucrose (MW=342 $\mathrm{g} / \mathrm{mol}$ ) in $5 \mathrm{mM}$ 4-(2-hydroxyethyl)-1piperazineethanesulfonic acid (HEPES) at $\mathrm{pH}=7.4$ at a final sucrose concentration of $195 \mathrm{mM}$. This washing buffer was used for three purposes: i) to dissolve all tested metabolites, ii) to electroform vesicles of all lipid chemistries, and iii) to wash away from the chip the extra-vesicle fluorescent probe CF. All tested metabolites [i.e. glycine (product number G7126 in Merck), alanine (A7627), leucine (L8000), aspartic acid (A9256), lysine (L5501), glutamine (G8540), tryptophan (T0254), glyceraldehyde (G5001), dihydroxyacetone (PHR1430), deoxyribose (31170), ribose (R7500), arabinose (A3256), glycerol (G9012), cytosine (C3506), uracil (U0750), adenine (A8626), guanine (G11950), urea (U5378), (2-aminoethyl)phosphonic acid (268674) and adenosine monophosphate (A1752), see Table S2], were dissolved in the washing buffer at a final concentration of $1 \mathrm{mM}$.

\section{Preparation of vesicles}

Giant unilamellar vesicles (simply referred as vesicles in the manuscript) were electroformed by using Vesicle Prep Pro (Nanion). The phospholipid of interest was weighed and dissolved in chloroform at a stock concentration of $10 \mathrm{mM}$. Next, $10 \mu \mathrm{L}$ of each phospholipid solution was spread using a pipette (Starlab) on the conductive side of an ITO-glass slide. The glass slide was then placed in a vacuum desiccator for $15 \mathrm{~min}$ to dry out the lipid solution. Meanwhile, the washing buffer was prepared as described above, and $495 \mu \mathrm{L}$ of this buffer was degassed and mixed with $5 \mu \mathrm{L}$ of CF stock solution $(10 \mathrm{mg} / \mathrm{mL})$ at a final CF concentration of 0.266 $\mathrm{mM}$. The ITO-slide coated by a dry lipid layer was then placed inside the Vesicle Prep Pro chamber and a greasy rubber O-ring was placed around the dry lipid layer. The area within the O-ring was then filled with 300 $\mu \mathrm{L}$ of $0.266 \mathrm{mM} \mathrm{CF}$ solution. A second ITO-slide was placed above the O-ring with its conductive side facing downwards and the Vesicle Prep Pro chamber was then closed. The electroformation process was carried out using a different electroformation protocol for each lipid or lipid mixture employed (see Table S3). Briefly, the electroformation process was performed in three steps. The rise step: the AC voltage was linearly increased peak to peak $(p-p)$ from $0 \mathrm{~V}$ till the maximum chosen value (see Table S3). The main step: the voltage was kept constant for the chosen period of time (see Table S3). The fall step: the voltage was decreased linearly to $0 \mathrm{~V}$. For lipids 9 and 10 none of the protocols employed yielded mechanically stable vesicles. For lipid 6 none of the employed protocols allowed vesicle electroformation possibly because this lipid forms non-lamellar structures of either a cubic or hexagonal fashion (44). The protocols for the electroformation of vesicles made of lipids 7, 8 and 11 were adapted from (52). The final fluorescent vesicle suspension consisted of fluorescent 
vesicles (because of the embedded CF molecules) and free CF molecules in the washing buffer. This suspension was collected from the ITO-slide surface using a pipette, placed in an Eppendorf microcentrifuge tube for storage at $4{ }^{\circ} \mathrm{C}$ and used within five days.

\section{Design and fabrication of the microfluidic chip}

The microfluidic chip was designed in AutoCAD and consisted of two inlets connected to a main chamber which splits into four parallel channels, containing 60 hydrodynamic traps each (henceforth coves) and further connected to a single outlet with an overall footprint of $0.8 \mathrm{~mm} \times 1.3 \mathrm{~mm}$ (Figure 1). Each cove was designed by drawing two concentric circles of radii 15 and $35 \mu \mathrm{m}$, respectively. A straight vertical line was drawn on the diameter of the outer circle from top to bottom and the left sides of the two semi-circles were deleted. A second $10 \mu \mathrm{m}$ long vertical line was drawn within the inner semi-circle, followed by two horizontal semi-infinite lines starting at the vertices of this line. The whole area included in these three lines was then deleted, yielding the final design of each cove. In first approximation, this shape resembles a cove with an opening in the middle. This design was printed on a film mask (Microlithography Services Ltd) and used for fabricating a mold of the fluidic chip via photolithography $(53,54)$, as detailed in the accompanying methodology on protocols.io (https://www.protocols.io/private/3AAE4162B49711EA84120A58A9FEAC2A). Briefly, a $20 \mu \mathrm{m}$ thick layer of SU-8 3025 (Microchem) was deposited via spin coating (6,000 r.p.m. for 30 s) on a silicon wafer (Si-Mat Silicon Materials), baked at $95^{\circ} \mathrm{C}$ for $10 \mathrm{~min}$, exposed to ultraviolet light $\left(1.3 \mathrm{~s}, 400 \mathrm{~nm}, 50 \mathrm{mWcm}^{-2}\right)$ through the film mask above and developed after baking at $65^{\circ} \mathrm{C}$ for $2 \mathrm{~min}$ and $95^{\circ} \mathrm{C}$ for $3 \mathrm{~min}$. Polydimethylsiloxane (PDMS) replica of this device were produced via soft lithography as previously described(55). Briefly, a 10:1 (base:curing agent) PDMS mixture (SYLGARD ${ }^{\mathrm{TM}} 184$ Silicone Elastomer Kit, Dow) was cast on the mold, degassed for $30 \mathrm{~min}$ and cured at $70^{\circ} \mathrm{C}$ for $2 \mathrm{~h}$ in an oven. The cured PDMS was peeled from the mold and fluidic accesses were created by using a $1.5 \mathrm{~mm}$ biopsy punch (Integra Miltex). Oxygen plasma treatment was used to irreversibly seal the PDMS chip on a glass coverslip (10 s exposure to $30 \mathrm{~W}$ plasma power, Plasma etcher, Diener Electronic $\mathrm{GmbH}$ ). We note that, compared to microfluidic devices previously employed for single-cell confinement (56), our approach relies on a single height device that can be fabricated in a single step without the use of dedicated photolithography equipment and can be easily carried out by users who might not be familiar with the technical aspects of microfabrication.

\section{Microfluidic permeability assay}

$15 \mu \mathrm{L}$ of washing buffer was injected in the microfluidic chip from the buffer inlet (Figure 1) using a pipette until the buffer spilled out from both the metabolite inlet and the outlet. Next, the metabolite inlet was temporarily sealed with a $1 \mathrm{~cm} \times 1 \mathrm{~cm}$ piece of Scotch Magic ${ }^{\mathrm{TM}}$ tape and $15 \mu \mathrm{L}$ of the fluorescence vesicle suspension was pipetted into the chip via the washing buffer inlet. Once a cove had been occupied by a vesicle, the amount of fluid entering the cove was reduced (56) but still allowed molecular diffusion in the cove, which was crucial for the permeability assay. Using the tape prevented the fluorescent vesicle suspension from entering into the metabolite inlet. The chip was then transferred under the microscope. A $1 \mathrm{~mL}$ plastic syringe (Becton Dickinson) was filled with the washing buffer and a second $1 \mathrm{~mL}$ syringe was filled with a $1 \mathrm{mM}$ solution of the metabolite under investigation. Both syringes were connected to 23-gauge needles (Becton Dickinson) and Masterflex Transfer Tygon tubing with $0.5 \mathrm{~mm}$ inner and $1.5 \mathrm{~mm}$ outer diameter (Cole-Parmer Instrument). Next, the syringes were connected to a Nemesys pump controlled via the QmixElements software (Centoni). The tape was then removed from the metabolite inlet and the tubing containing the metabolite solution under 
investigation was inserted into the metabolite inlet while the metabolite solution was flowing at a rate of 0.5 $\mu \mathrm{L} / \mathrm{h}$. Next, the tubing containing the washing buffer was inserted into the washing buffer inlet while the buffer solution was flowing at a rate of $5 \mu \mathrm{L} / \mathrm{h}$. These flow rates were then simultaneously increased at steps of 0.5 $\mu \mathrm{L} / \mathrm{h}$ every $10 \mathrm{~s}$ up to $2 \mu \mathrm{L} / \mathrm{h}$ for the metabolite solution and at steps of $5 \mu \mathrm{L} / \mathrm{h}$ every $10 \mathrm{~s}$ up to $25 \mu \mathrm{L} / \mathrm{h}$ for the buffer solution (Figure 1A). These maximal flow rates were kept constant for 20 min to remove any free CF molecules from the microfluidic environment while the fluorescent vesicles remained confined in the coves (Figure 1B). Flowing the metabolite solution at a low rate (i.e. $2 \mu \mathrm{L} / \mathrm{h}$ ) prevented the accumulation of free CF molecules in the metabolite inlet but did not affect the permeability assay since we obtained similar permeability traits in the absence or presence of this low flow rate. Next, the metabolite solution under investigation was delivered to the fluorescent vesicles in the coves by reducing the flow rate of the washing buffer from $25 \mu \mathrm{L} / \mathrm{h}$ to $1 \mu \mathrm{L} / \mathrm{h}$ and by increasing the flow rate of the metabolite solution from $2 \mu \mathrm{L} / \mathrm{h}$ to $25 \mu \mathrm{L} / \mathrm{h}$ (Figure 1C). Next, the chip was visually inspected by using a $20 \times 0.85$ N.A. oil-immersion objective mounted on an inverted epifluorescence microscope (Olympus IX73) equipped with a sCMOS camera (Zyla 4.2, Andor, used at an exposure time of $0.1 \mathrm{~s}$ ), a blue LED (CoolLED pE300white, used at $30 \%$ of its intensity) and a FITC filter. This setup allowed us to simultaneously image 12 coves, therefore the area of the chip that contained the higher number of vesicles trapped in the coves was chosen. An image of such an area was acquired immediately after increasing the metabolite solution flow rate and then at intervals of $30 \mathrm{~s}$ for $3 \mathrm{~min}$. All experiments were performed at an intra- and extra-vesicle $\mathrm{pH}$ of 7.4 , since $\mathrm{pH}$ changes are known to affect $\mathrm{CF}$ fluorescence intensity (57). For each membrane mimic in Table S1, in order to account for the impact of both the delivery of the washing buffer solution and photobleaching on the intra-vesicle CF fluorescence signal, we performed separate control assays by connecting the metabolite inlet to a syringe containing the washing buffer solution instead of the metabolite solution. Apart from this modification, these control assays were carried out following the protocol described above for the microfluidic permeability assays. The intra-vesicle CF fluorescence consistently linearly decreased during the delivery of the washing buffer for all membrane mimics investigated and this information was used to provide a background signal for the corresponding microfluidic permeability assays (see Image and data analysis section below).

\section{Image and data analysis}

Images of the fluorescent vesicles for each membrane mimic trapped in the microfluidic coves during the 3 min delivery of each metabolite were imported in Image J to produce a temporal image stack (58) and analysed as follows to extract quantitative comparative information about the permeability traits of each membrane mimic to each metabolite. Only images of unilamellar vesicles were retained for analysis, whereas images of multilamellar vesicles were not taken forward. To obtain the single-vesicle temporal dependence of intravesicle fluorescence, for each image and each time point, a circle was drawn around each vesicle. A second circle of identical dimensions was drawn in an area $10 \mu \mathrm{m}$ to the left of the vesicle. This allowed us to extract the mean CF fluorescence of each vesicle and the background around it at each time point, respectively. The background fluorescence was subtracted from the corresponding intra-vesicle fluorescence for each vesicle and each time point. Next, for each vesicle from all the background subtracted values, the initial intra-vesicle fluorescence value (at $t=0$ ) was subtracted. Next, these values were corrected to account for the impact of both the delivery of the washing buffer solution and photobleaching on the intra-vesicle CF fluorescence signal. To do so a correction factor was calculated from the microfluidic control assay data sets (see below), multiplied 
by each time value and added to the corresponding intra-vesicle fluorescence value (after the background and initial fluorescence value subtractions above). To obtain a correction factor for each membrane mimic, first we applied the image analysis protocol above to obtain the single-vesicle temporal dependence of intra-vesicle fluorescence values during the delivery of the washing buffer and subtract from these values the corresponding background and initial intra-vesicle fluorescence value. Second, we averaged these temporal dependences of corrected single-vesicle fluorescence values to obtain a mean temporal dependence for each membrane mimic during the delivery of the washing buffer. Finally, we fitted this mean temporal dependence to a linear regression with the intercept forced to zero and obtained the slope of the linear fluorescence decrease for each membrane mimic. These slope values were used as correction factors to calculate the permeability of each membrane mimic to each metabolite as described above. For some of the membrane mimic and metabolite pairs we noticed a minority of outliers (i.e. vesicles that became either much brighter or dimmer with respect to the majority of vesicles investigated during metabolite delivery, see for example Figure 2B). This heterogeneity is common when investigating membrane permeability both in vitro(59) and in vivo(41, 60). The permeability of each membrane mimic to each metabolite was measured in three independent experiments. Throughout the manuscript $\mathrm{N}$ indicates the number of single vesicles investigated for each membrane mimic and metabolite pair. $\mathrm{N}$ varies across different metabolite-experiments investigated due to the fact that the number of vesicles trapped within the microfluidic coves of the chosen chip area can vary. However, care has been taken to obtain the same $\mathrm{N}$ for each metabolite-experiment across the different membrane mimics comparisons to ensure reliable statistical comparisons. In order to do so, when a statistical comparison was to be made between data sets of different $\mathrm{N}$, corrected intra-vesicle fluorescence values were randomly selected from the data set that contained the higher N. Such statistical comparisons were carried out via unpaired t-tests with Welch's correction between the distributions of corrected intra-vesicle fluorescence values at $\mathrm{t}=3 \mathrm{~min}$ for the different membrane mimics. ${ }^{* * * *}$ indicates a $\mathrm{p}$-value $<0.0001,{ }^{* * *}$ indicates a p-value $<0.001,{ }^{*}$ indicates a p-value $<0.01,{ }^{*}$ indicates a p-value $<0.05$, n.s. indicates a p-value $>0.05$. All permeability data and statistical comparisons were carried out and plotted using GraphPad Prism 9.

\section{In silico identification of prokaryotic transporter proteins}

To characterize the transporter repertoires of bacterial and archaeal species in silico, we first assembled a collection of prokaryotic reference proteomes from UniProt (release 2021_03 (51)). To minimize taxonomic redundancy, a single proteome was selected per genus based on BUSCO completeness scores (61), resulting in 3,044 bacterial and 244 archaeal proteomes. Data derived from environmental or metagenomic bacterial isolates were excluded due to the absence of morphological information. To comprehensively identify transporter homologs, profile hidden Markov models (HMMs) derived from TCDB (Transporter Classification Database) protein families (termed tcDoms, downloaded 2 June 2021) were used to search each proteome using HMMER v3.1b2 $\left(E<10^{-5}\right.$, incE $<10^{-5}$, domE $\left.<10^{-5}\right)(51,62)$. If multiple HMMs identified the same predicted protein, the protein was assigned to the family with the lower E-value. To improve the sensitivity of the HMMs, the hits from the initial HMM search were aligned using MAFFT v7.471 (-auto), trimmed with a gapthreshold of $10 \%$ using trimAl v1.4, and the resulting alignments were used to generate new HMMs $(51,63)$. Using the second iteration HMMs, another search was conducted as above, producing the final set of identified proteins. To reduce the potential effects of taxon sampling and dataset bias, prokaryotic taxa were grouped into their respective orders based on NCBI Taxonomy classifications (64). Transporter abundance was then 
interpreted as the median number of transporters assigned to a given TCDB family, normalized by the total number of proteins encoded by each taxa, across each order. The resulting distribution was visualized in $\mathrm{R}$ v4.0.2 and hierarchical clustering was done using euclidean distances and the Ward.D2 clustering method implemented by pheatmap (https://mran.microsoft.com/snapshot/2018-0831/web/packages/pheatmap/pheatmap.pdf). The differential abundance of individual transporter families was assessed by comparing archaeal and monoderm transporter abundances (given their morphological similarities) using Wilcoxon tests after Bonferroni correction.

To identify putative archaeal transporter families undetected by the TCDB HMMs, we clustered archaeal proteomes into protein families using the Markov clustering algorithm $(I=1.4)$ based on pairwise BLASTp searches conducted using Diamond v2.0.9.147 $\left(\mathrm{E}<10^{-5}\right.$, query coverage $>50 \%$, -max-target-seqs $=10^{5}$, --more-sensitive search option) $(65,66)$. Transmembrane (TM) domains were then predicted for each archaeal protein using Phobius and the median number of TM domains was determined for each protein family (67). Those families with representation in at least five archaeal species and with a median of at least four TM domains were identified and annotated using eggNOG mapper v2.1.2 (68). TM domain-containing protein families were classified as putative transporters if they were annotated with PFAM domains associated with transporter function (e.g., all transporter and channel-associated domains) (69). Bacterial homologs of archaeal protein families were detected using Diamond BLASTp by searching the bacterial reference proteomes with each protein family $\left(E<10^{-20}\right.$, query coverage $>50 \%$ ). To compare $\mathrm{TM}$ domain lengths and amino acid composition in Archaea and Bacteria, TM domains were predicted from archaeal and bacterial proteins using Phobius and their length and composition was assessed using BioPython (https://academic.oup.com/bioinformatics/article/25/11/1422/330687?login=true).

\section{Author Contributions}

SP and TAR designed the research. UL and ZK performed the experiments. NATI performed in silico evolutionary analysis of transporter families. UL, ZK, NATI, DSM, AES, TAR and SP analysed and discussed the data. UL, TR and SP wrote the paper. All authors read and approved the final manuscript.

\section{Acknowledgments}

U.L., Z.K. and D.S.M. were supported via a Gordon and Betty Moore Foundation Marine Microbiology Initiative grant (GBMF5514) awarded to T.A.R., A.E.S. and S.P.. U.L. was also supported through a BBSRC responsive mode grant (BB/V008021/1) awarded to S.P.. N.A.T.I. was supported by a Junior Research Fellowship from Merton College, University of Oxford. S.P. and T.A.R.'s work in this area was also supported by a Marie Skłodowska-Curie project SINGEK (H2020-MSCA-ITN-2015-675752).

\section{References}

1. C. R. Woese, G. E. Fox, Phylogenetic structure of the prokaryotic domain: The primary kingdoms. Proc. Natl. Acad. Sci. U. S. A. 74, 5088-5090 (1977).

2. C. R. Woese, O. Kandler, M. L. Wheelis, Towards a natural system of organisms: Proposal for the domains Archaea, Bacteria, and Eucarya. Proc. Natl. Acad. Sci. U. S. A. 87, 4576-4579 (1990).

3. S. L. Baldauf, J. D. Palmer, W. F. Doolittle, The root of the universal tree and the origin of eukaryotes based on elongation factor phylogeny. Proc. Natl. Acad. Sci. U. S. A. 93, 7749-7754 (1996). 
4. J. R. Brown, W. F. Doolittle, Root of the universal tree of life based on ancient aminoacyl-tRNA synthetase gene duplications. Proc. Natl. Acad. Sci. U. S. A. 92, 2441-2445 (1995).

5. G. P. Fournier, J. P. Gogarten, Rooting the ribosomal tree of life. Mol. Biol. Evol. 27, 1792-1801 (2010).

6. G. A. Coleman, R. D. Pancost, T. A. Williams, GBE Investigating the Origins of Membrane Phospholipid Biosynthesis Genes Using Outgroup-Free Rooting. Genome Biol. Evol. 11, 883-898 (2019).

7. T. J. Williams, M. Allen, B. Tschitschko, R. Cavicchioli, Glycerol metabolism of haloarchaea. Environ. Microbiol. 19, 864-877 (2017).

8. T. A. Williams, P. G. Foster, C. J. Cox, T. M. Embley, An archaeal origin of eukaryotes supports only two primary domains of life. Nature 504, 231-236 (2013).

9. S. Pagliara, et al., Study of optical properties of electrospun light-emitting polymer fibers. Superlattices Microstruct. 47, 145-149 (2010).

10. J. Esko, T. Doering, C. Raetz, Eubacteria and Archaea, editors. E. of G. In: Varki A, Cummings RD, Esko JD, et al., Ed., 2nd Ed. (Cold Spring Harbor (NY): Cold Spring Harbor Laboratory Press, 2009).

11. Z. Kelman, J. Hurwitz, Structural lessons in DNA replication from. Nat. Struct. Biol. 10, 148 (2003).

12. F. Mattiroli, et al., Structure of histone-based chromatin in Archaea. Science (80-. ). 357, 609-612 (2017).

13. J. Lombard, P. López-García, D. Moreira, The early evolution of lipid membranes and the three domains of life. Nat. Rev. Microbiol. 10, 507-515 (2012).

14. V. Sojo, Why the Lipid Divide? Membrane Proteins as Drivers of the Split between the Lipids of the Three Domains of Life. BioEssays 41, 1-6 (2019).

15. D. Segré, D. Ben-Eli, D. W. Deamer, D. Lancet, The Lipid World. Orig. Life Evol. Biosph. 31, 119-145 (2001).

16. T. M. Mccollom, G. Ritter, B. R. T. Simoneit, Lipid synthesis under hydrothermal conditions by Fischer-Tropsch-type reactions. Orig. Life Evol. Biosph. 29, 153-166 (1999).

17. M. M. Hanczyc, S. M. Fujikawa, J. W. Szostak, Experimental Models of Primitive Cellular Compartments: Encapsulation, Growth, and Division. Science (80-. ). 302, 618-622 (2003).

18. L. Eme, A. Spang, J. Lombard, C. W. Stairs, T. J. G. Ettema, Archaea and the origin of eukaryotes. Nat. Rev. Microbiol. 15, 711-723 (2017).

19. S. Jain, A. Caforio, A. J. M. Driessen, Biosynthesis of archaeal membrane ether lipids. Front. Microbiol. 5, 1-16 (2014).

20. V. Sojo, A. Pomiankowski, N. Lane, A Bioenergetic Basis for Membrane Divergence in Archaea and Bacteria. PLoS Biol. 12, e1001926 (2014).

21. K. Adamala, J. W. Szostak, Competition between model protocells driven by an encapsulated catalyst. Nat. Chem. 5, 495-501 (2013).

22. I. Budin, J. W. Szostak, Physical effects underlying the transition from primitive to modern cell membranes. Proc. Natl. Acad. Sci. U. S. A. 108, 5249-5254 (2011).

23. H. Shimada, A. Yamagishi, Stability of heterochiral hybrid membrane made of bacterial sn -G3P lipids and archaeal sn -G1P lipids. Biochemistry 50, 4114-4120 (2011).

24. J. C. Blain, J. W. Szostak, Progress toward synthetic cells. Annu. Rev. Biochem. 83, 615-640 (2014).

25. S. F. Jordan, E. Nee, N. Lane, Isoprenoids enhance the stability of fatty acid membranes at the 
emergence of life potentially leading to an early lipid divide. Interface Focus 9 (2019).

26. H. J. Morowitz, B. Heinz, D. W. Deamer, The chemical logic of a minimum protocell. Orig. Life Evol. Biosph. 18, 281-287 (1988).

27. A. Khvorova, Y. G. Kwak, M. Tamkun, I. Majerfeld, M. Yarus, RNAs that bind and change the permeability of phospholipid membranes. Proc. Natl. Acad. Sci. U. S. A. 96, 10649-10654 (1999).

28. J. C. Mathai, S. Tristram-Nagle, J. F. Nagle, M. L. Zeidel, Structural determinants of water permeability through the lipid membrane. J. Gen. Physiol. 131, 69-76 (2008).

29. D. L. Valentine, Adaptations to energy stress dictate the ecology and evolution of the Archaea. Nat. Rev. Microbiol. 5, 1070-1077 (2007).

30. S. S. Mansy, et al., Template-directed synthesis of a genetic polymer in a model protocell. Nature 454, 122-125 (2008).

31. J. C. Mathai, G. D. Sprott, M. L. Zeidel, Molecular Mechanisms of Water and Solute Transport across Archaebacterial Lipid Membranes *. J. Biol. Chem. 276, 27266-27271 (2001).

32. K. Yamauchi, K. Doi, Y. Yoshida, M. Kinoshita, Archaebacterial lipids: highly proton-impermeable membranes from 1,2-diphytanyl-sn-glycero-3-phosphocoline. BBA - Biomembr. 1146, 178-182 (1993).

33. M. E. Bozdaganyan, et al., Proton leakage across lipid bilayers: Oxygen atoms of phospholipid ester linkers align water molecules into transmembrane water wires. Biochim. Biophys. Acta - Bioenerg. 1860, 439-451 (2019).

34. I. K. Jarsch, F. Daste, J. L. Gallop, Membrane curvature in cell biology: An integration of molecular mechanisms. J. Cell Biol. 214, 375-387 (2016).

35. K. S. Ramamurthi, R. Losick, Negative membrane curvature as a cue for subcellular localization of a bacterial protein. Proc. Natl. Acad. Sci. U. S. A. 106, 13541-13545 (2009).

36. J. Ude, et al., Outer membrane permeability: Antimicrobials and diverse nutrients bypass porins in Pseudomonas aeruginosa. Proc. Natl. Acad. Sci. U. S. A. 118, 1-8 (2021).

37. J. P. Schrum, T. F. Zhu, J. W. Szostak, The origins of cellular life. Cold Spring Harb. Perspect. Biol. 2, 1-15 (2010).

38. T. Cavalier-smith, Obcells as Proto-Organisms : Membrane Heredity, Lithophosphorylation, and the Origins of the Genetic Code, the First Cells, and Photosynthesis. J. Mol. Evol. 53, 555-595 (2001).

39. M. G. Sacerdote, J. W. Szostak, Semipermeable lipid bilayers exhibit diastereoselectivity favoring ribose. Proc. Natl. Acad. Sci. U. S. A. 102, 6004-6008 (2005).

40. M. Orsi, W. E. Sanderson, J. W. Essex, Permeability of small molecules through a lipid bilayer: A multiscale simulation study. J. Phys. Chem. B 113, 12019-12029 (2009).

41. U. Lapinska, G. Glover, P. Capilla-lasheras, A. J. Young, S. Pagliara, Bacterial ageing in the absence of external stressors. Philos. Trans. R. Soc. B Biol. Sci. 374, 20180442 (2019).

42. J. Hu, W. G. Cochrane, A. X. Jones, D. G. Blackmond, B. M. Paegel, Chiral lipid bilayers are enantioselectively permeable. Nat. Chem. 13, 786-791 (2021).

43. D. Poger, B. Caron, A. E. Mark, Effect of methyl-branched fatty acids on the structure of lipid bilayers. J. Phys. Chem. B 118, 13838-13848 (2014).

44. S. Kara, et al., Diphytanoyl lipids as model systems for studying membrane-active peptides. Biochim. Biophys. Acta - Biomembr. 1859, 1828-1837 (2017).

45. S. Paula, A. G. Volkov, D. W. Deamer, Permeation of halide anions through phospholipid bilayers 
occurs by the solubility-diffusion mechanism. Biophys. J. 74, 319-327 (1998).

46. F. J. Elling, et al., Chemotaxonomic characterisation of the thaumarchaeal lipidome. Environ. Microbiol. 19, 2681-2700 (2017).

47. T. Bauersachs, K. Weidenbach, R. A. Schmitz, L. Schwark, Distribution of glycerol ether lipids in halophilic, methanogenic and hyperthermophilic archaea. Org. Geochem. 83-84, 101-108 (2015).

48. J. Cama, C. Chimerel, S. Pagliara, A. Javer, U. F. Keyser, A label-free microfluidic assay to quantitatively study antibiotic diffusion through lipid membranes. Lab Chip 14, 2303-8 (2014).

49. A. Blicher, K. Wodzinska, M. Fidorra, M. Winterhalter, T. Heimburg, The temperature dependence of lipid membrane permeability, its quantized nature, and the influence of anesthetics. Biophys. J. 96, 4581-4591 (2009).

50. A. Caforio, et al., Converting Escherichia coli into an archaebacterium with a hybrid heterochiral membrane. Proc. Natl. Acad. Sci. U. S. A. 115, 3704-3709 (2018).

51. M. H. Saier, et al., The transporter classification database (TCDB): 2021 update. Nucleic Acids Res. 49, D461-D467 (2021).

52. D. Balleza, A. B. Garcia-Arribas, J. Sot, K. Ruiz-Mirazo, F. M. Goñi, Ether- Versus ester-linked phospholipid bilayers containing either linear or branched apolar chains. Biophys. J. 107, 1364-1374 (2014).

53. E. Locatelli, et al., Single-File Escape of Colloidal Particles from Microfluidic Channels. Phys. Rev. Lett. 117, 038001 (2016).

54. U. Lapinska, et al., Gradient-free determination of isoelectric points of proteins on chip. Phys. Chem. Chem. Phys. 19, 23060-23067 (2017).

55. S. L. Dettmer, U. F. Keyser, S. Pagliara, Local characterization of hindered Brownian motion by using digital video microscopy and 3D particle tracking. Rev. Sci. Instrum. 85 (2014).

56. D. Di Carlo, N. Aghdam, L. P. Lee, Single-cell enzyme concentrations, kinetics, and inhibition analysis using high-density hydrodynamic cell isolation arrays. Anal. Chem. 78, 4925-4930 (2006).

57. R. Sjöback, J. Nygren, M. Kubista, Absorption and fluorescence properties of fluorescein. Spectrochim. Acta Part A Mol. Spectrosc. 51 (1995).

58. O. Goode, et al., Persister Escherichia coli Cells Have a Lower Intracellular pH than Susceptible Cells but Maintain Their pH in Response to. MBio 12, e00909-21 (2021).

59. J. Cama, K. Al Nahas, M. Fletcher, K. Hammond, G. Maxim, An ultrasensitive microfluidic approach reveals correlations between the physico-chemical and biological activity of experimental peptide antibiotics. bioRxiv (2021) https:/doi.org/10.1101/2021.09.08.459503.

60. M. R. L. Stone, et al., Fluorescent macrolide probes - synthesis and use in evaluation of bacterial resistance. RSC Chem. Biol. 1, 395-404 (2020).

61. F. A. Simão, R. M. Waterhouse, P. loannidis, E. V. Kriventseva, E. M. Zdobnov, BUSCO: Assessing genome assembly and annotation completeness with single-copy orthologs. Bioinformatics 31, 32103212 (2015).

62. J. Mistry, R. D. Finn, S. R. Eddy, A. Bateman, M. Punta, Challenges in homology search: HMMER3 and convergent evolution of coiled-coil regions. Nucleic Acids Res. 41 (2013).

63. S. Capella-Gutiérrez, J. M. Silla-Martínez, T. Gabaldón, trimAl: A tool for automated alignment trimming in large-scale phylogenetic analyses. Bioinformatics 25, 1972-1973 (2009).

64. S. Federhen, The NCBI Taxonomy database. Nucleic Acids Res. 40, 136-143 (2012). 
65. B. Buchfink, K. Reuter, H. G. Drost, Sensitive protein alignments at tree-of-life scale using DIAMOND. Nat. Methods 18, 366-368 (2021).

66. A. J. Enright, S. Van Dongen, C. A. Ouzounis, An efficient algorithm for large-scale detection of protein families. Nucleic Acids Res. 30, 1575-1584 (2002).

67. L. Käll, A. Krogh, E. L. L. Sonnhammer, A combined transmembrane topology and signal peptide prediction method. J. Mol. Biol. 338, 1027-1036 (2004).

68. J. Huerta-Cepas, et al., Fast genome-wide functional annotation through orthology assignment by eggNOG-mapper. Mol. Biol. Evol. 34, 2115-2122 (2017).

69. S. El-Gebali, et al., The Pfam protein families database in 2019. Nucleic Acids Res. 47, D427-D432 (2019). 
Figures

\section{A}

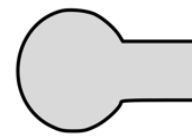

Washing buffer inlet

Flow rate: $25 \mu \mathrm{l} / \mathrm{h}$

B

Metabolite inlet
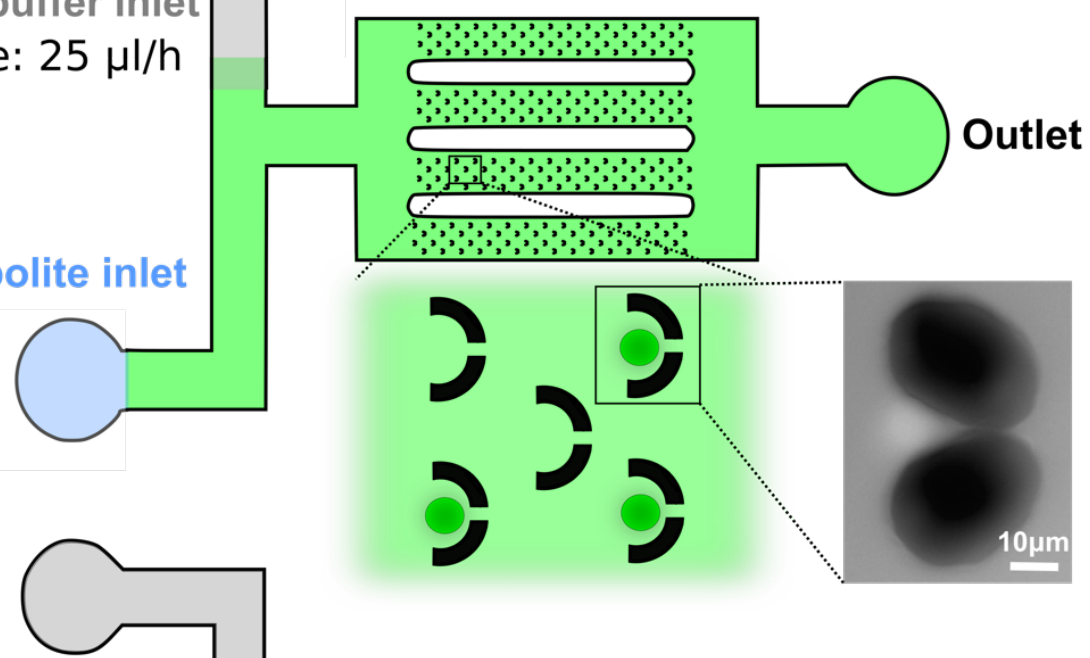

\section{Washing buffer inlet}

Metabolite inlet

Flow rate: $25 \mu \mathrm{l} / \mathrm{h}$
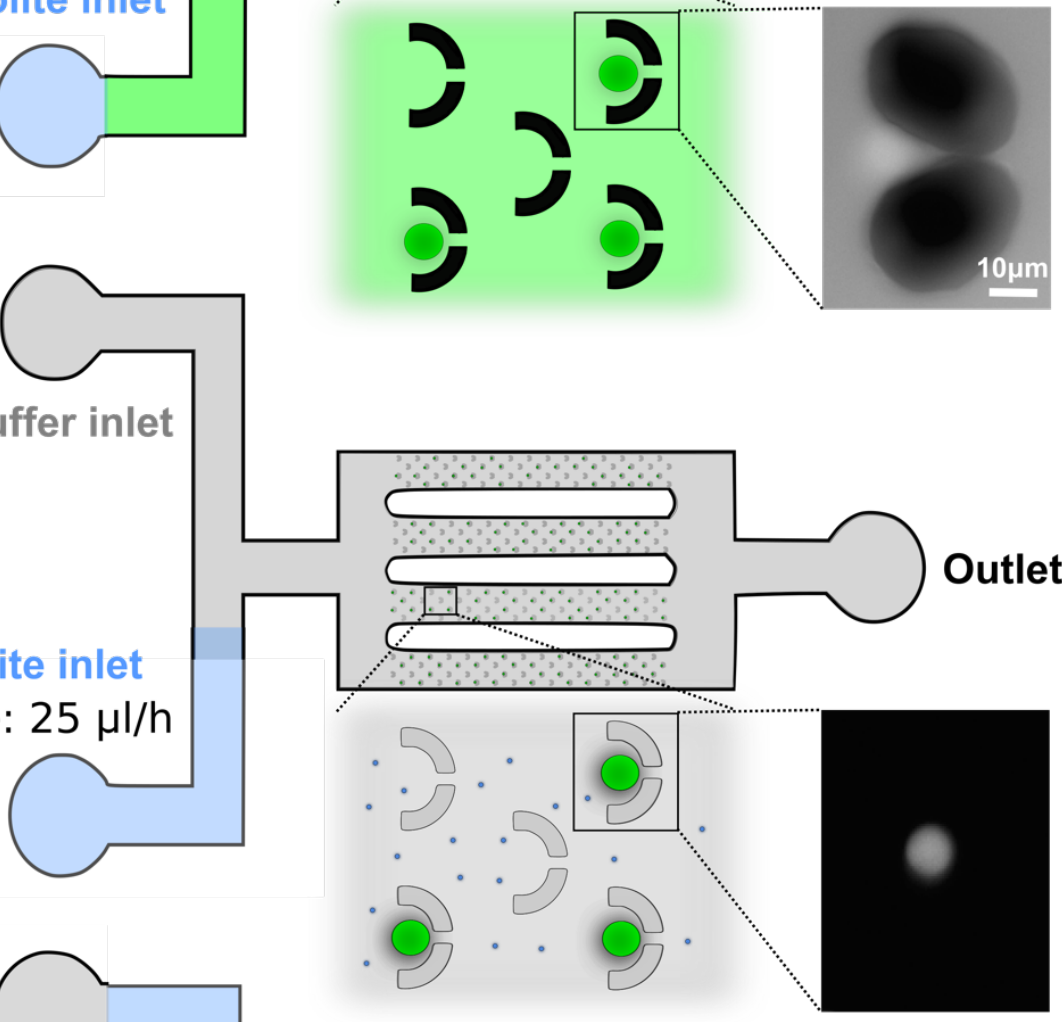

C

\section{Washing buffer inlet}

Metabolite inlet

Flow rate: $25 \mu \mathrm{l} / \mathrm{h}$
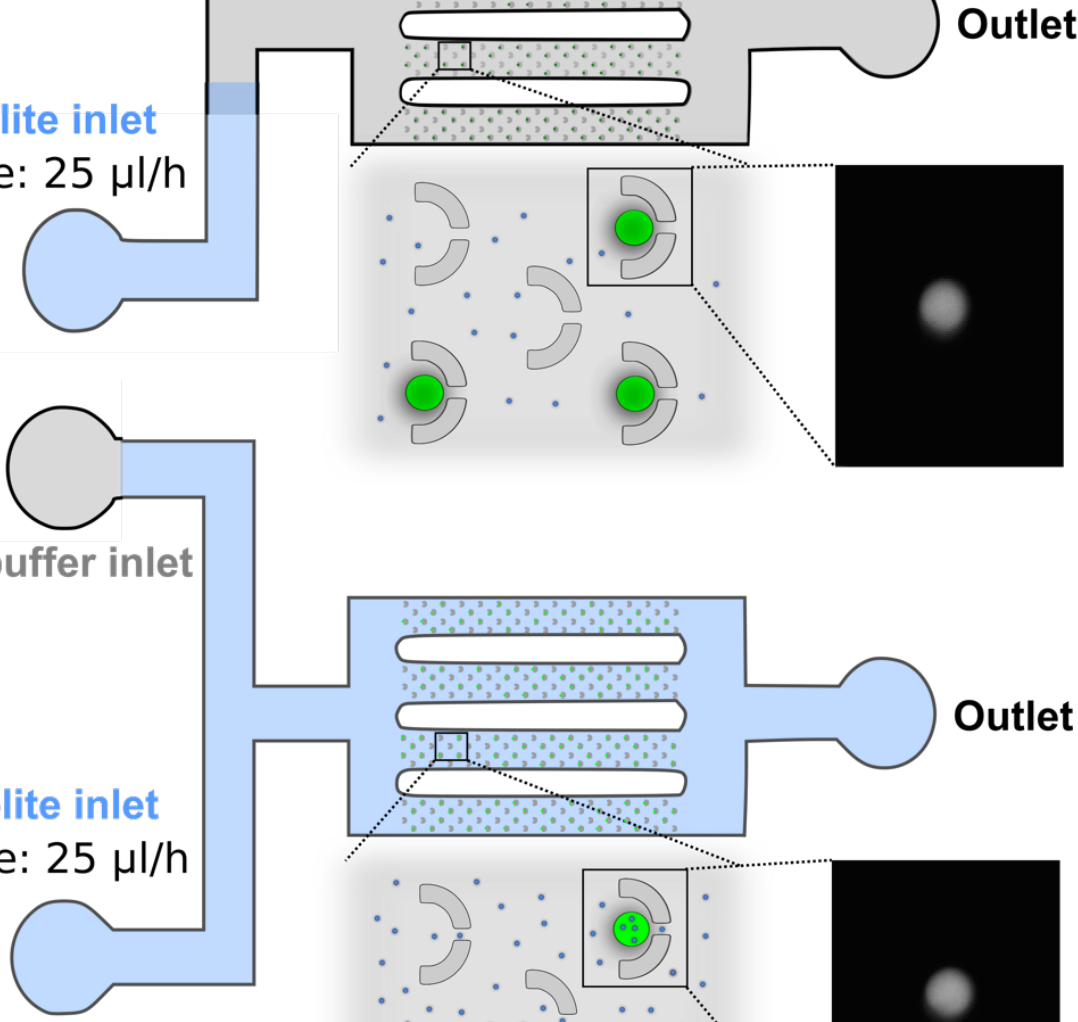

Outlet

Time (min)

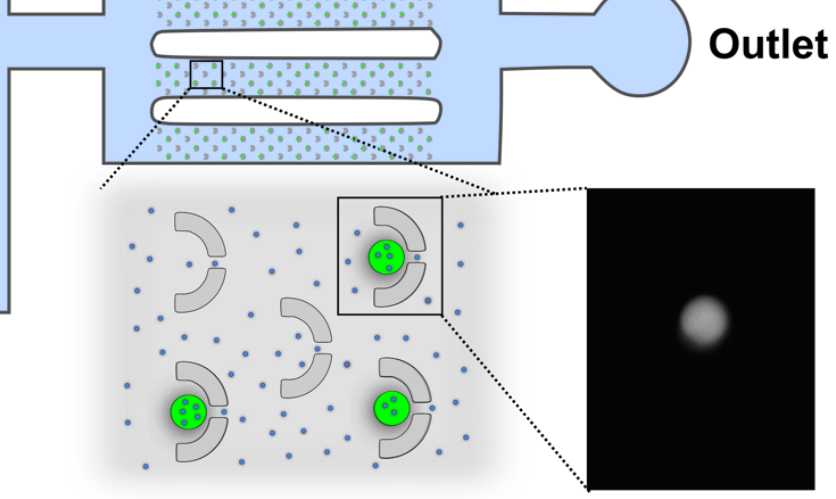

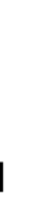


are removed from the microfluidic environment around the vesicles by applying a flow rate of $25 \mu \mathrm{L} / \mathrm{h}$ from the washing buffer inlet for 20 minutes. b) At $t=0$ min vesicles appear bright on a dark background as shown in the micrograph. The metabolite (small blue circles) under investigation is delivered to the vesicles by applying a flow rate of $25 \mu \mathrm{L} / \mathrm{h}$ from the metabolite inlet for 3 minutes. c) The metabolite accumulates within the vesicles if their membrane is permeable to the metabolite, thus the intra-vesicle fluorescence at $t=3$ min is higher compared to that measured at $\mathrm{t}=0 \mathrm{~min}$ as shown in the micrographs.
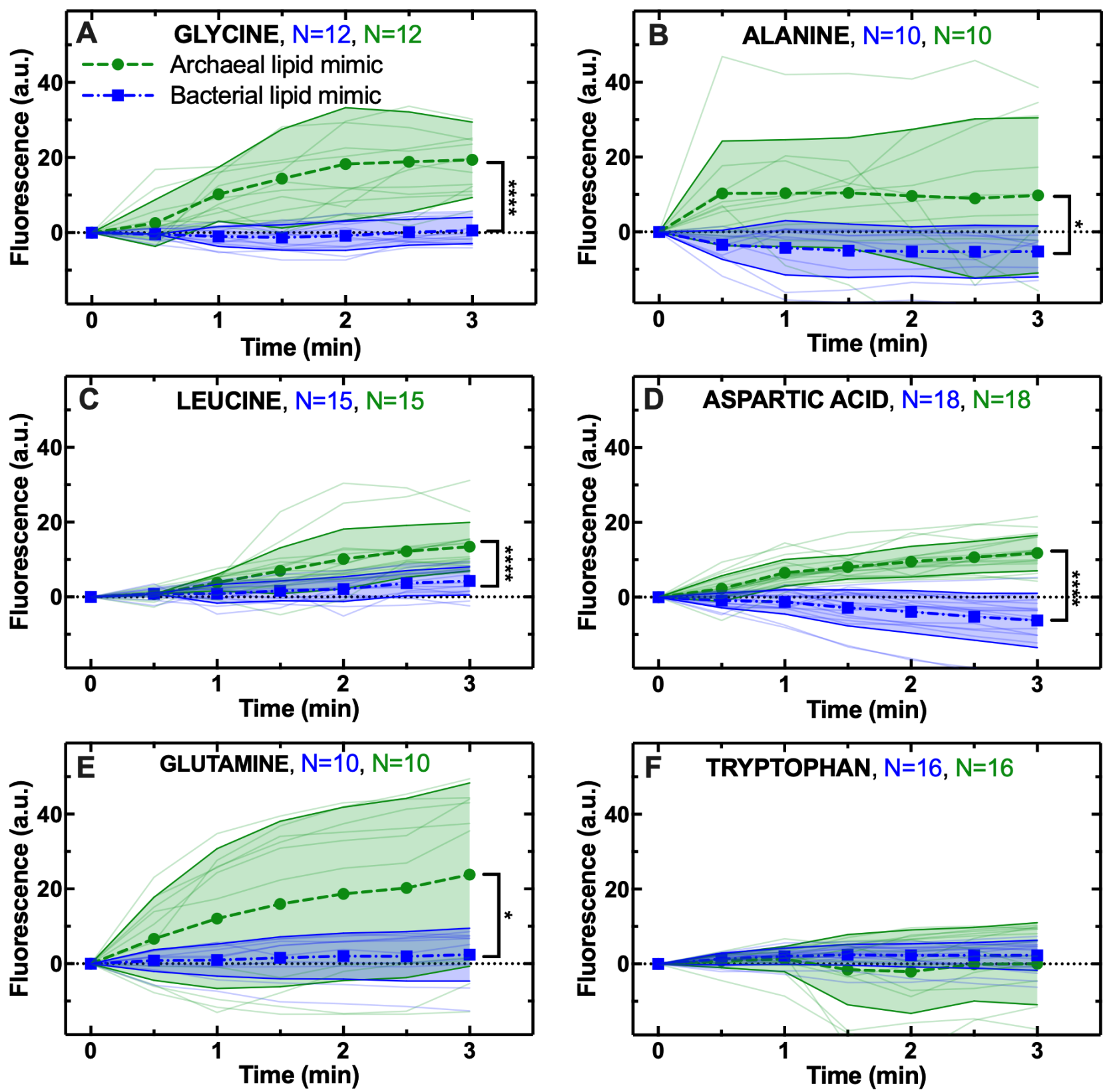

Figure 2. The archaeal membrane mimic is consistently more permeable to amino acids compared to the bacterial membrane mimic. Temporal dependence of average CF fluorescence in archaeal (green circles) and bacterial (blue squares) membrane mimics during the exposure to $1 \mathrm{mM}$ a) glycine, b) alanine, c) leucine, d) aspartic acid, e) glutamine or $\mathbf{f}$ ) tryptophan delivered to the microfluidic coves. Mean (symbols) and standard deviation (shaded areas) were calculated from at least 10 single-vesicle measurements (solid lines) across 3 independent experiments. $\mathrm{N}$ is the number of single vesicles investigated for each amino acid and each membrane mimic (green and blue for the archaeal and bacterial membrane mimic, respectively). $\mathrm{N}$ varies 
across different metabolite-experiments investigated due to technical constraints (see Methods). However, care has been taken to obtain the same $\mathrm{N}$ for each metabolite-experiment across the two different membrane mimics to ensure reliable statistical comparisons. Such comparisons have been carried out via unpaired t-tests with Welch's correction between the distributions of CF fluorescence values at $t=3$ min for the archaeal and bacterial membrane mimics. ${ }^{* * *}$ : $p$-value $<0.0001,{ }^{*}$ : $p$-value $<0.05$. The lipids used for creating the archaeal and bacterial membrane mimics are lipids 1 and 2, respectively, in Table S1.
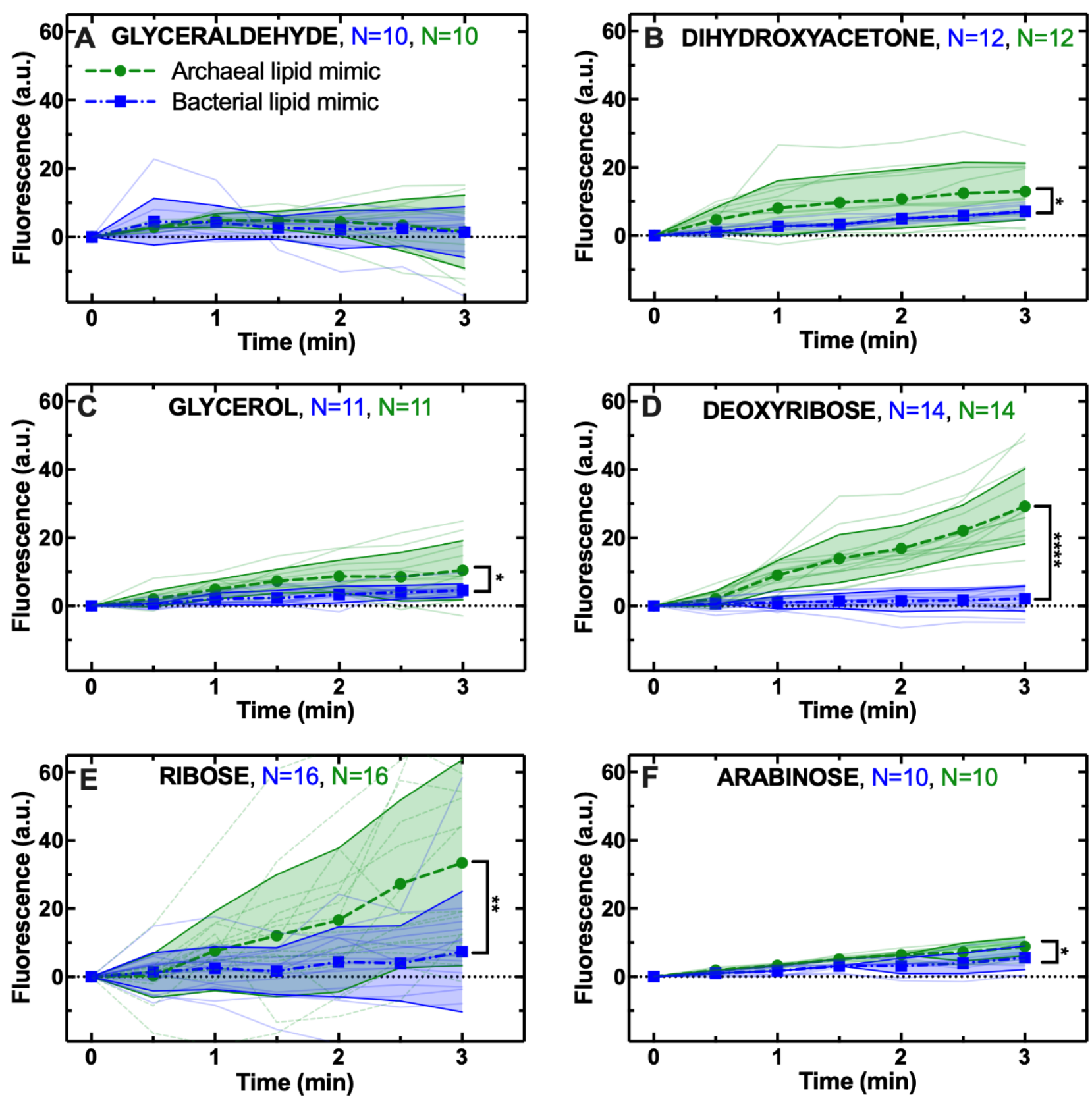

Figure 3. The archaeal membrane mimic is more permeable to sugar constituents of RNA and DNA compared to the bacterial membrane mimic. Temporal dependence of average CF fluorescence in archaeal (green circles) and bacterial (blue squares) membrane mimics during the exposure to $1 \mathrm{mM}$ a) glyceraldehyde, b) dihydroxyacetone, c) glycerol, d) deoxyribose, e) ribose or $\mathbf{f}$ ) arabinose delivered to the microfluidic coves at $\mathrm{t}=0$. Mean (symbols) and standard deviation (shaded areas) were calculated from at least 10 single-vesicle measurements (solid lines) across 3 independent experiments. $\mathrm{N}$ is the number of single vesicles investigated 
for each sugar and each membrane mimic (green and blue for the archaeal and bacterial membrane mimic, respectively). $\mathrm{N}$ varies across different metabolite-experiments investigated due to technical constraints (see Methods). However, care has been taken to obtain the same $\mathrm{N}$ for each metabolite-experiment across the two different membrane mimics to ensure reliable statistical comparisons. Such comparisons have been carried out via unpaired t-tests with Welch's correction between the distributions of CF fluorescence values at $\mathrm{t}=3 \mathrm{~min}$ for the archaeal and bacterial membrane mimics. ${ }^{* * * *}$ : $p$-value $<0.0001,{ }^{* *}$ : $p$-value $<0.01,{ }^{*}$ : p-value $<0.05$. The lipids used for creating the archaeal and bacterial membrane mimics are lipids 1 and 2, respectively, in Table S1.
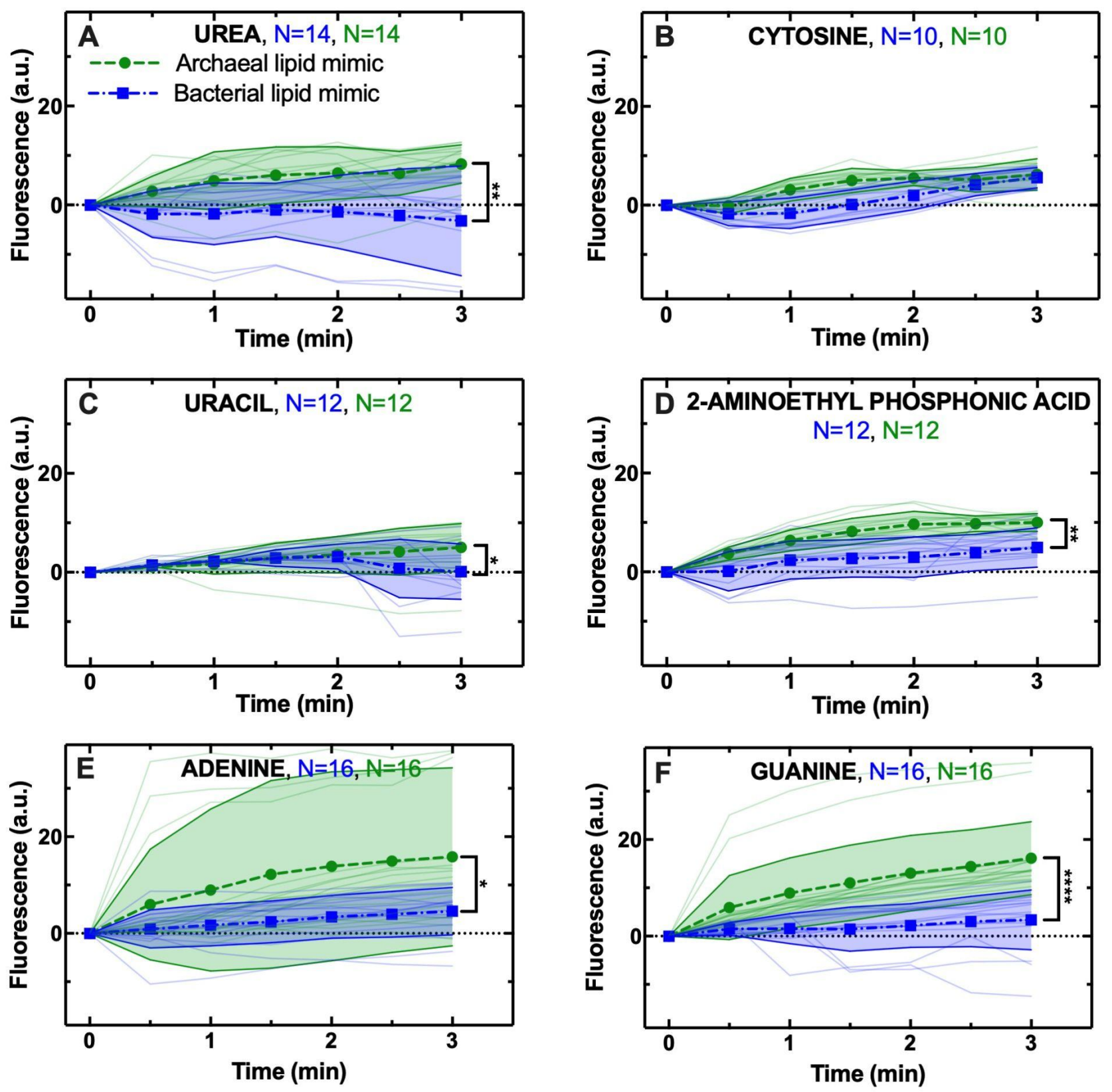

Figure 4. The archaeal membrane mimic is more permeable to nucleobases, urea and phosphonate compared to the bacterial membrane. Temporal dependence of average CF fluorescence in archaeal (green circles) and bacterial (blue squares) membrane mimics during exposure to $1 \mathrm{mM}$ a) urea, b) cytosine, c) uracil, d) 2-aminoethyl phosphonic acid, e) adenine or $\mathbf{f}$ ) guanine delivered to the microfluidic coves at $\mathrm{t}=0$. Mean 
(symbols) and standard deviation (shaded areas) were calculated from at least 10 single-vesicle measurements (solid lines) across 3 independent experiments. $N$ is the number of single vesicles investigated for each metabolite and each membrane mimic (green and blue for the archaeal and bacterial membrane mimic, respectively). $\mathrm{N}$ varies across different metabolite-experiments due to technical constraints (see Methods). However, care has been taken to obtain the same $\mathrm{N}$ for each metabolite-experiment across the two different membrane mimics to ensure reliable statistical comparisons. Such comparisons have been carried out via unpaired t-tests with Welch's correction between the distributions of CF fluorescence values at $\mathrm{t}=3 \mathrm{~min}$ for the archaeal and bacterial membrane mimics. ${ }^{* * *}$ : $p$-value $<0.0001,{ }^{* *}$ : $p$-value $<0.01,{ }^{*}$ : p-value $<0.05$. The lipids used for creating the archaeal and bacterial membrane mimics are lipids 1 and 2, respectively, in Table S1. 

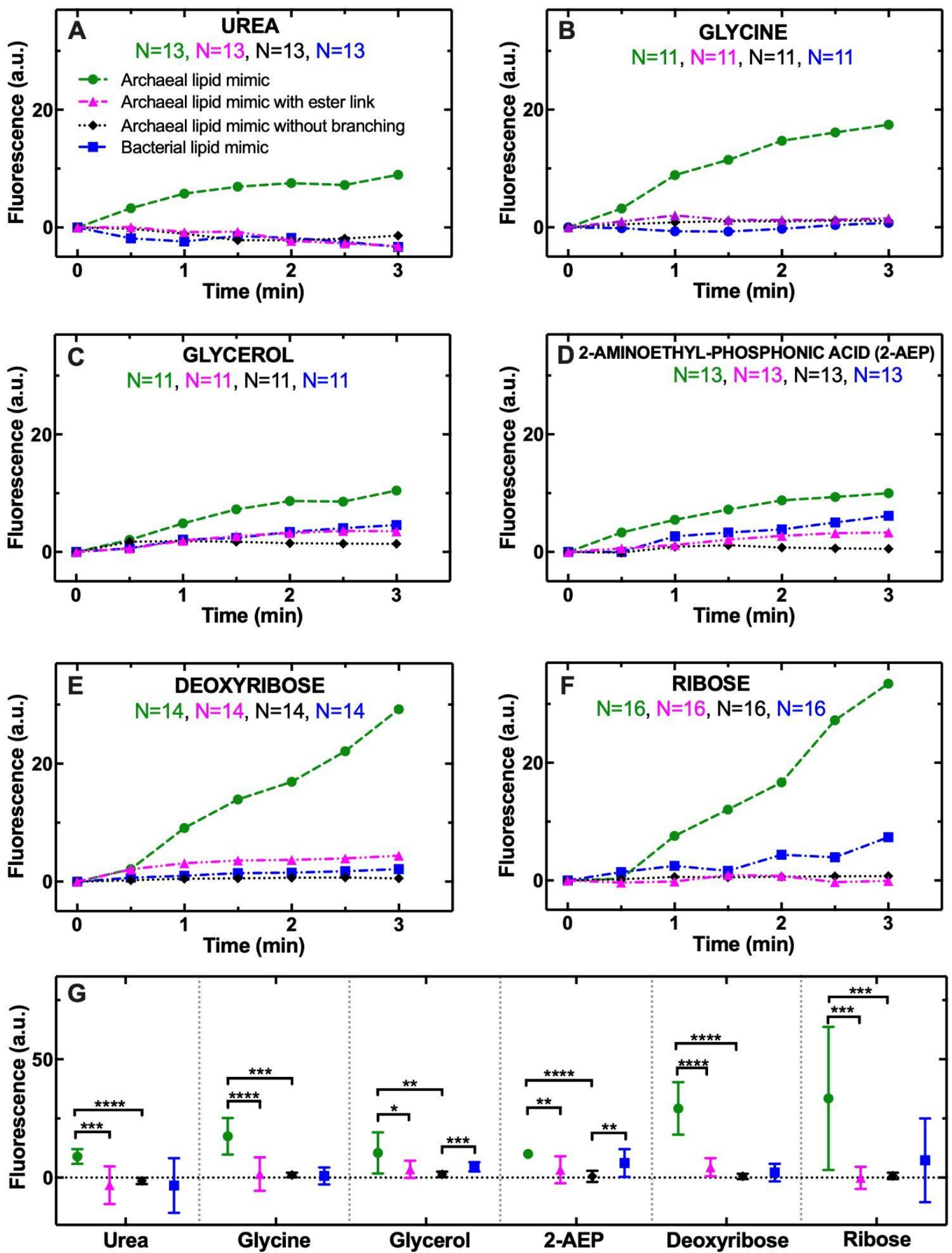

Figure 5. Chain branching and ether linkage have profound implications on archaeal membrane permeability to small metabolites. Temporal dependence of average CF fluorescence in archaeal membrane mimics without branching (black diamonds) or in archaeal membrane mimics with an ester in place of an ether link (magenta triangles) during the exposure to $1 \mathrm{mM}$ a) urea, b) glycine, c) glycerol, d) 2-aminoethyl phosphonic acid, $\mathbf{e}$ ) deoxyribose or $\mathbf{f}$ ) ribose delivered to the microfluidic coves at $t=0$. These substrates were 
chosen as previous analyses demonstrate that these had key differences in permeability characteristics. Data for the archaeal and bacterial membrane mimics are reproduced from Figures 2, 3 and 4 for comparison purposes. Means (symbols) were calculated from at least 10 single-vesicle measurements across 3 independent experiments. Due to the large overlaps between the measurements obtained with the different membrane mimics, standard deviations and single-vesicle measurements are not reported for clarity, with a summary of the statistical analysis reported below. Such comparisons have been carried out via unpaired ttests with Welch's correction between the distributions of CF fluorescence values at $t=3$ min for each membrane mimic pair and are reported in g). ${ }^{* * * *}$ : p-value $<0.0001,{ }^{* * *}$ : $p$-value $<0.001,{ }^{* *}$ : p-value $<0.01,{ }^{*}$ : $\mathrm{p}$-value $<0.05$. $\mathrm{N}$ is the number of single vesicles investigated for each metabolite and each membrane mimic. $\mathrm{N}$ varies across different metabolite-experiments investigated due to technical constraints (see Methods). However, care has been taken to obtain the same $\mathrm{N}$ for each metabolite-experiment across the two different membrane mimics to ensure reliable statistical comparisons. The lipids used for creating the archaeal membrane mimic, the bacterial membrane mimic, the archaeal membrane mimic without tail branching and the archaeal membrane mimic with an ester (in place of an ether) link are lipids 1, 2, 4 and 5, respectively, in Table S1. 

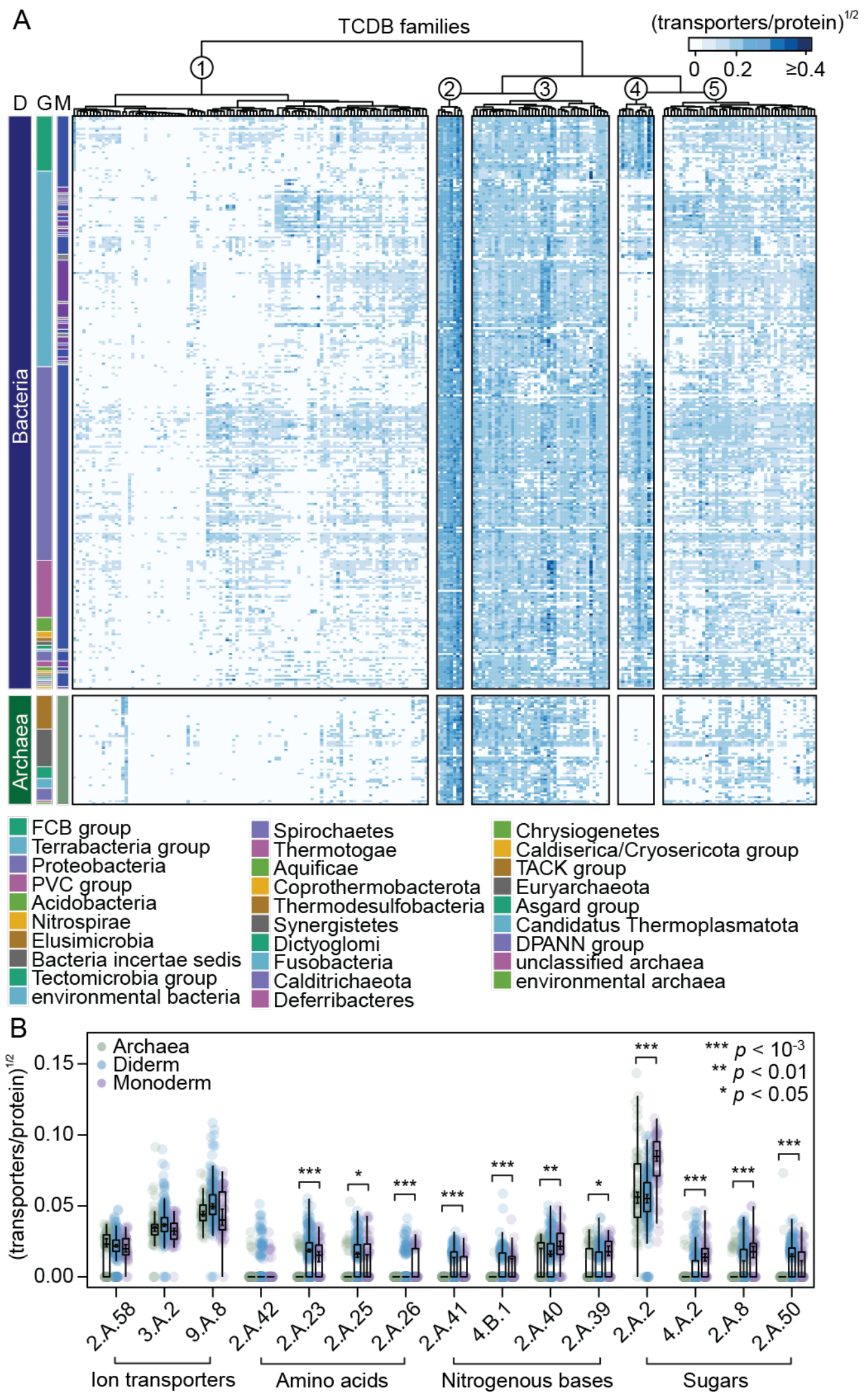

Figure 6. Archaea feature reduced transporter repertoires relative to Bacteria. a) Heatmap showing transporter repertoires in Archaea and Bacteria, where each row and column represent a prokaryotic order and TCDB transporter family, respectively. Heat map values represent the median number of transporters normalized by total protein count for every taxon across each prokaryotic order after a square root 
transformation. The domain (D), group (G), and membrane morphology (M: where blue, purple, and grey, represent diderms, monoderms, and unknown membrane morphology, respectively) of each order is noted. TCDB families were grouped by hierarchical clustering based on transporter abundance using euclidean distances and the Ward.D2 clustering method. Taxonomy was based on NCBI Taxonomy classifications. b) Individual comparisons of transporter families in Archaea, monoderms, and diderms. All transporter families predicted to translocate amino acids, nitrogenous bases, and sugars are shown, alongside three exemplary ion transporter families from Cluster 2. Comparisons were made using Wilcoxon tests and Bonferroni corrected $p$ values are shown. To assess taxon sampling bias, bootstrap-estimated $90 \%$ confidence intervals $(n=1,000)$ of the median are displayed over each boxplot median based on random sampling of prokaryotic orders with replacement. 


\section{SUPPLEMENTARY FIGURES}

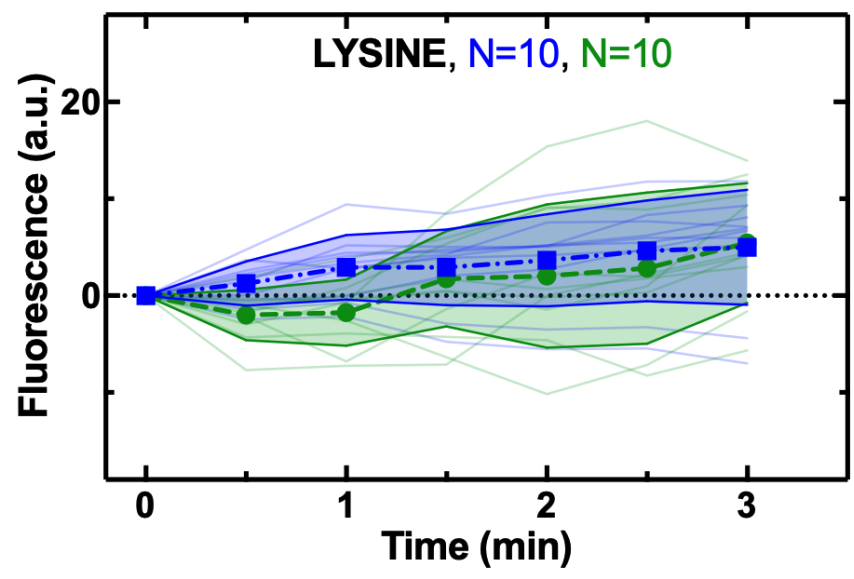

Figure S1. The archaeal and bacterial membranes are similarly permeable to the positively charged amino acid lysine. Temporal dependence of average CF fluorescence in archaeal (green circles) and bacterial (blue squares) membrane mimics during the exposure to $1 \mathrm{mM}$ lysine delivered to the microfluidic coves at $\mathrm{t}=0$. Mean (symbols) and standard deviation (shaded areas) were calculated from 10 single-vesicle measurements (solid lines) for each membrane mimic across 3 independent experiments. The lipids used for creating the archaeal and bacterial membrane mimics are lipids 1 and 2, respectively, in Table S1.

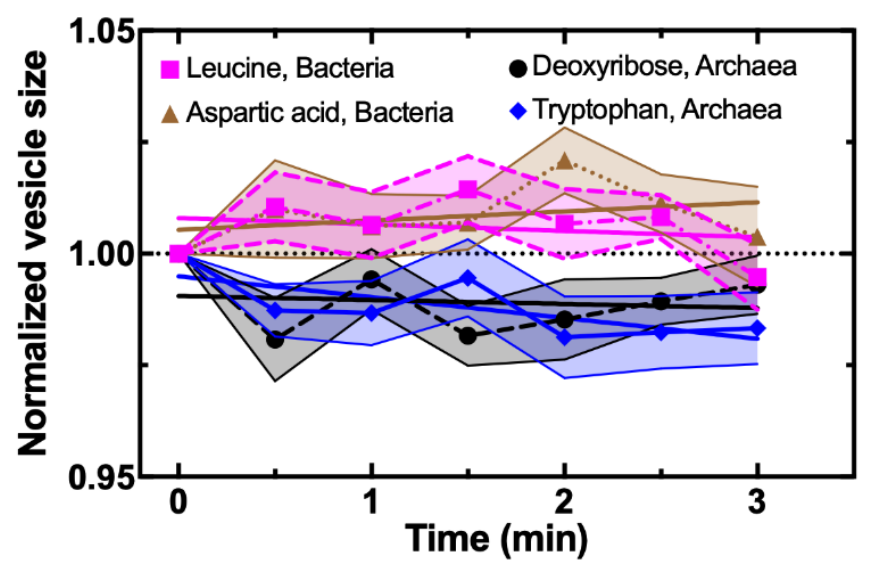

Figure S2. Metabolite permeation does not significantly affect vesicle size. Temporal dependence of the average vesicle size during the delivery of leucine or aspartic acid to vesicles mimicking bacterial membranes and deoxyribose or tryptophan to vesicles mimicking archaeal membranes. Mean (symbols) and standard deviation (shaded areas) were calculated by firstly normalising all values of each vesicle size to the size at $t=0$ and then averaging over the values measured on $\mathrm{N}=15$ vesicles for each metabolite and membrane mimic. Corresponding permeability data for each metabolite and membrane mimic are presented in Figures 2 and 3. No significant change in vesicle size was measured during the delivery of deoxyribose or tryptophan to the archaeal membrane mimic ( $p$-value $=0.77$ and 0.06 , respectively) or during the delivery of aspartic acid or leucine to the bacterial membrane mimic ( $p$-value $=0.47$ and 0.61 , respectively). Moreover, no significant change in vesicle size or shape was measured during the delivery of any of the metabolites in Figures 2-4. However, for clarity we report here only the temporal dependence of vesicle size during the delivery of the 
metabolites for which each membrane mimic was either most or least permeable. The lipids used for creating the archaeal and bacterial membrane mimics are lipids 1 and 2, respectively, in Table S1.
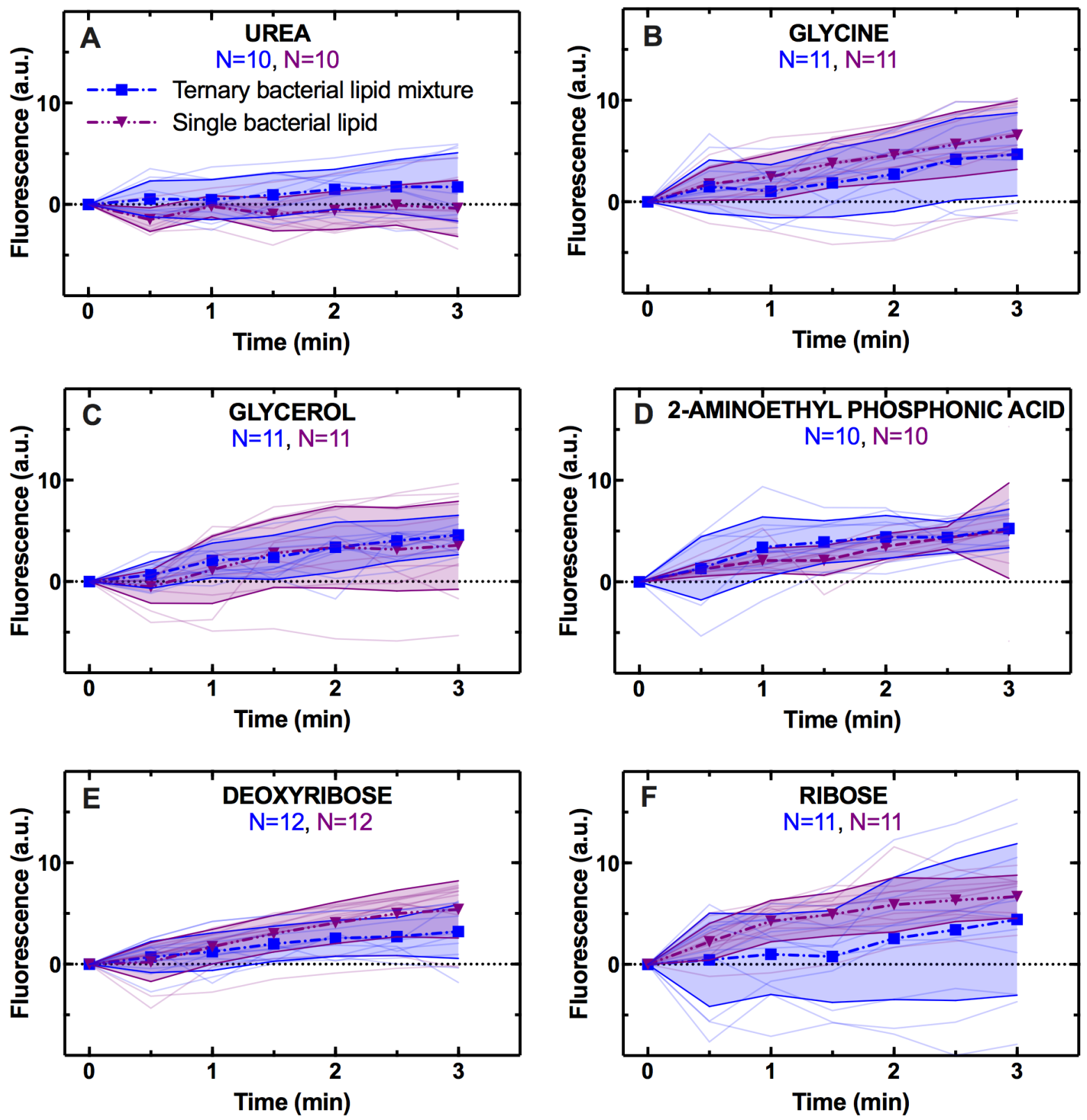

Figure S3. Mono- and ternary-lipid mixtures display similar permeability traits. Temporal dependence of average CF fluorescence in bacterial membrane mimics made of one lipid (purple triangles) or a ternary lipid mixture (blue squares) during the exposure to $1 \mathrm{mM}$ a) urea, b) glycine, c) glycerol, d) 2-aminoethyl phosphonic acid, e) deoxyribose or $\mathbf{f}$ ) ribose delivered to the microfluidic coves at $t=0$. Mean (symbols) and standard deviation (shaded areas) were calculated from at least 10 single-vesicle measurements (solid lines) across 3 independent experiments. $\mathrm{N}$ is the number of single vesicles investigated for each metabolite and each membrane mimic (blue and purple for the ternary and single lipid membrane mimic, respectively). $\mathrm{N}$ varies across different metabolite-experiments investigated due to technical constraints (see Methods). However, care has been taken to obtain the same $\mathrm{N}$ for each metabolite-experiment across the two different membrane mimics to ensure reliable statistical comparisons. Such comparisons have been carried out via unpaired t-tests with Welch's correction between the distributions of CF fluorescence values at $t=3$ min for the 
mono- and ternary-lipid mixture. The lipids used for creating the bacterial membrane mimics with ternary and single-lipid mixtures are lipids 2 and 5, respectively, in Table S1.
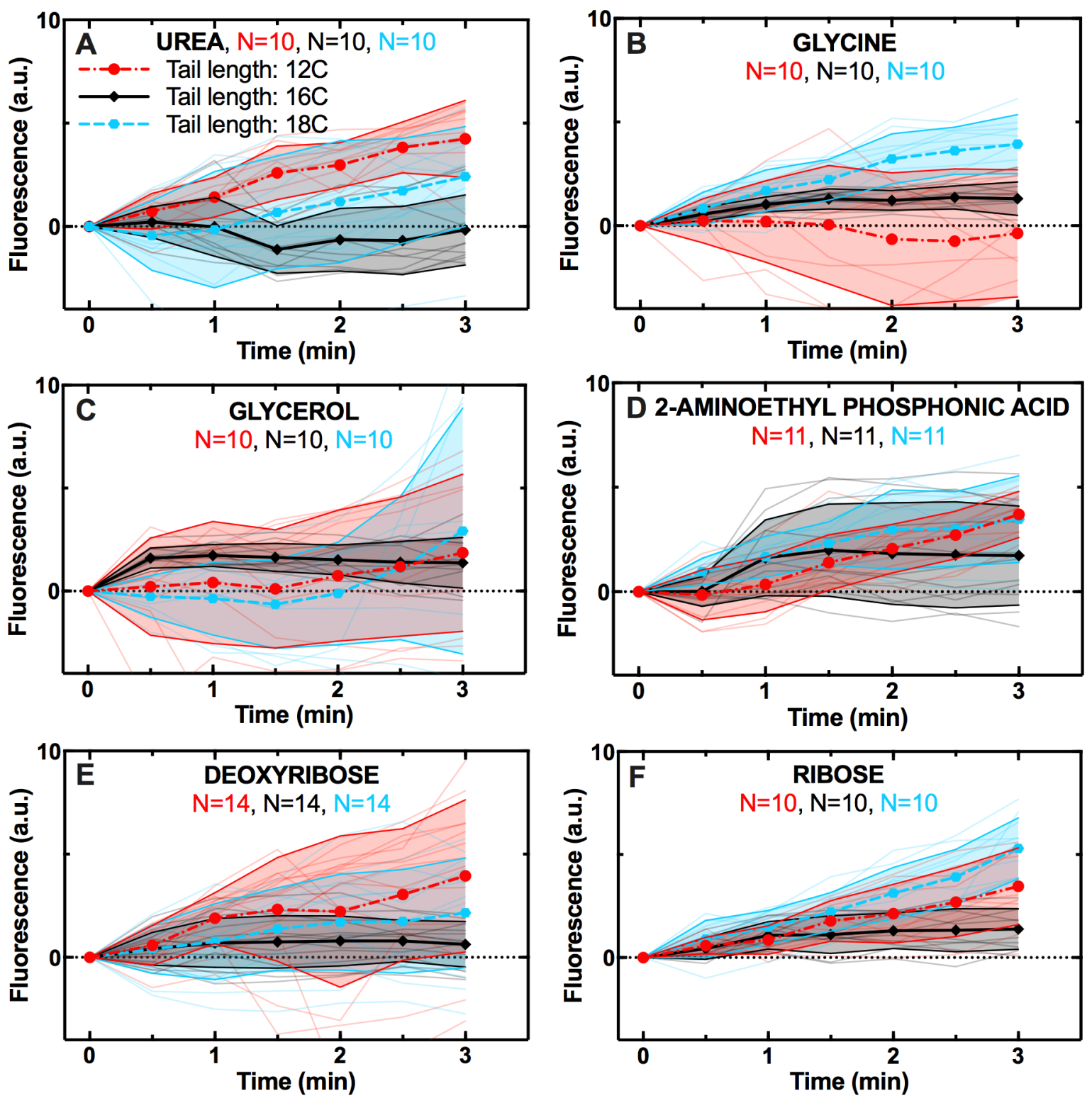

Figure S4. Archaeal phospholipid chain length does not have a significant impact on the permeability to core metabolites. Temporal dependence of average CF fluorescence in archaeal membrane mimics with tail length of 12 (red circles), 16 (black diamonds) or 18 carbons (blue hexagons) during the exposure to $1 \mathrm{mM}$ a) urea, b) glycine, c) glycerol, d) 2-aminoethyl phosphonic acid, e) deoxyribose or f) ribose delivered to the microfluidic coves at $\mathrm{t}=0$. Mean (symbols) and standard deviation (shaded areas) were calculated from at least 10 single-vesicle measurements (solid lines) across 3 independent experiments. $\mathrm{N}$ is the number of single vesicles investigated for each metabolite and each membrane mimic. $\mathrm{N}$ varies across different metaboliteexperiments investigated due to technical constraints (see Methods). However, care has been taken to obtain the same $\mathrm{N}$ for each metabolite-experiment across the two different membrane mimics to ensure reliable statistical comparisons. Such comparisons were carried out by evaluating the Pearson correlation coefficient between the average fluorescence value at $\mathrm{t}=3 \mathrm{~min}$ and the tail length. The measured coefficients were -0.58 , $0.95,0.52,-0.10,-0.69$ and 0.30 for urea, L-glycine, glycerol, 2-aminoethyl phosphonic acid, D-deoxyribose 
and D-ribose and not statistically significant for any of these metabolites. We could not investigate the permeability of archaeal membrane mimics with chain length of 14 carbons (lipid 10 in Table S1) because the transition temperature of these lipids (i.e. $24^{\circ} \mathrm{C}$ ) is very close to room temperature and vesicles easily burst during our permeability assays. Finally, we could not form vesicles using lipids with a chain length of 6 carbons (lipid 9 in Table S1) despite attempting different electroformation protocols (Table S3). The lipids used for creating the archaeal membrane mimics with tail length of 12,16 and 18 carbons are lipids 7,4 and 8 , respectively, in Table S1.
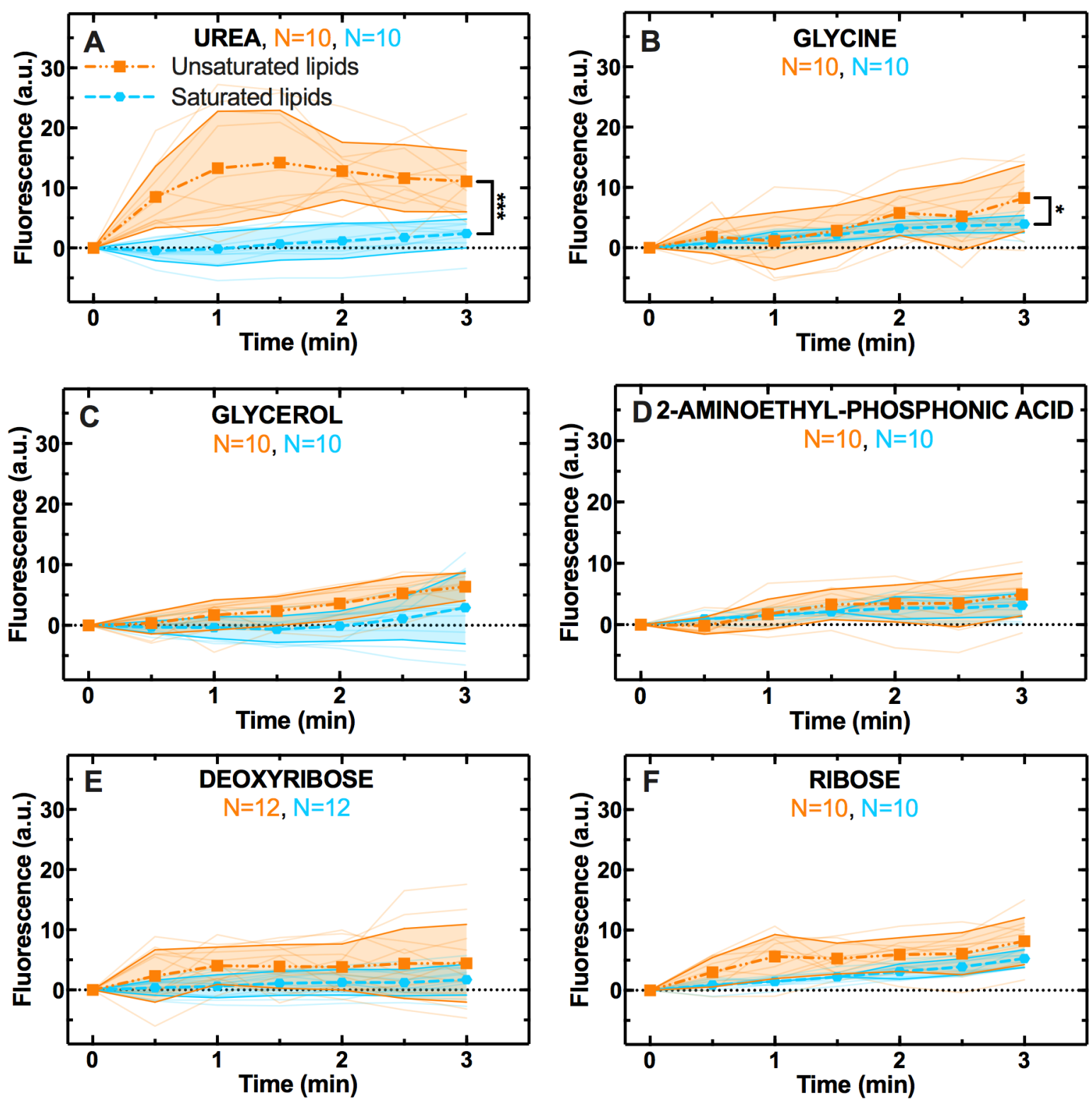

Figure S5. Unsaturated lipids favour permeability to urea and glycine. Temporal dependence of CF fluorescence in archaeal membrane mimics in the presence (orange squares) or absence of double bonds along the lipid chain (blue circles) during the exposure to $1 \mathrm{mM}$ a) urea, b) glycine, c) glycerol, d) 2-aminoethyl phosphonic acid, e) deoxyribose or $\mathbf{f}$ ) ribose delivered to the microfluidic coves at $t=0$. Mean (symbols) and standard deviation (shaded areas) were calculated from at least 10 single-vesicle measurements (solid lines) across 3 independent experiments. $N$ is the number of single vesicles investigated for each metabolite and each membrane mimic. $\mathrm{N}$ varies across different metabolite-experiments investigated due to technical constraints (see Methods). However, care has been taken to obtain the same $\mathrm{N}$ for each metabolite-experiment 
bioRxiv preprint doi: https://doi.org/10.1101/2021.10.12.464042; this version posted October 13, 2021. The copyright holder for this preprint (which was not certified by peer review) is the author/funder, who has granted bioRxiv a license to display the preprint in perpetuity. It is made available under aCC-BY 4.0 International license.

across the two different membrane mimics to ensure reliable statistical comparisons. Such comparisons have been carried out via unpaired t-tests with Welch's correction between the distributions of CF fluorescence values at $\mathrm{t}=3 \mathrm{~min}$ for the single and double bonding membrane mimics. ${ }^{* *}$ : $p$-value $<0.001,{ }^{*}$ : $p$-value $<0.05$. The lipids used for creating the archaeal membrane mimics with and without saturation are lipids 8 and 11 , respectively, in Table S1.
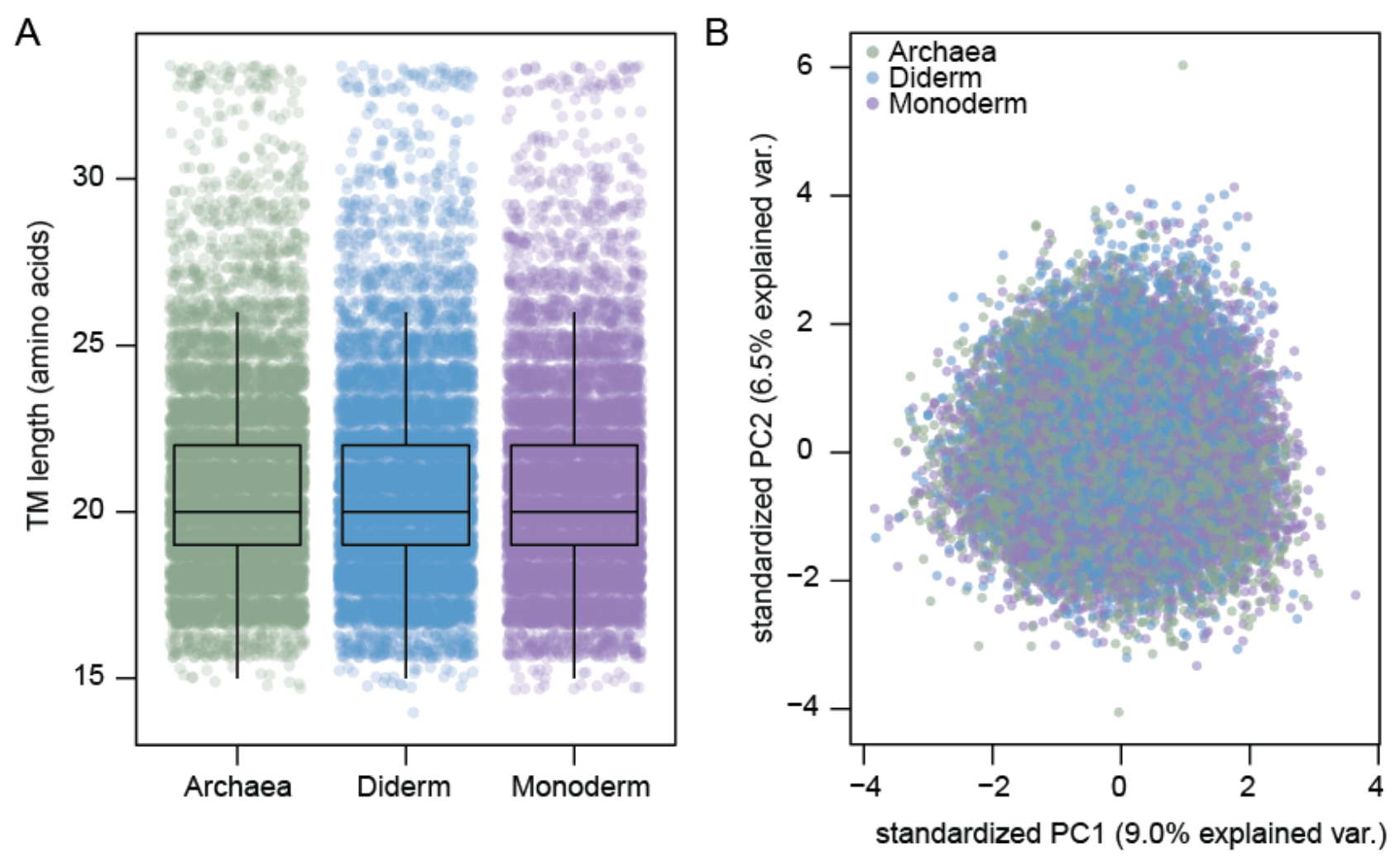

Figure S6. Archaeal and bacterial transmembrane domains are biochemically consistent. a) Comparisons between the length of transmembrane domains in Archaea and bacterial monoderms and diderms. Transmembrane domains were identified using Phobius. b) Principal component analyses based on the amino acid compositions of archaeal and bacterial transmembrane domains. In both analyses, archaeal and bacterial transmembrane domains were randomly subsampled to equivalent sample sizes $(n=10,000)$. 
bioRxiv preprint doi: https://doi.org/10.1101/2021.10.12.464042; this version posted October $13,2021$. The copyright holder for this

preprint (which was not certified by peer review) is the author/funder, who has granted bioRxiv a license to display the preprint in perpetuity. It is made available under aCC-BY 4.0 International license.

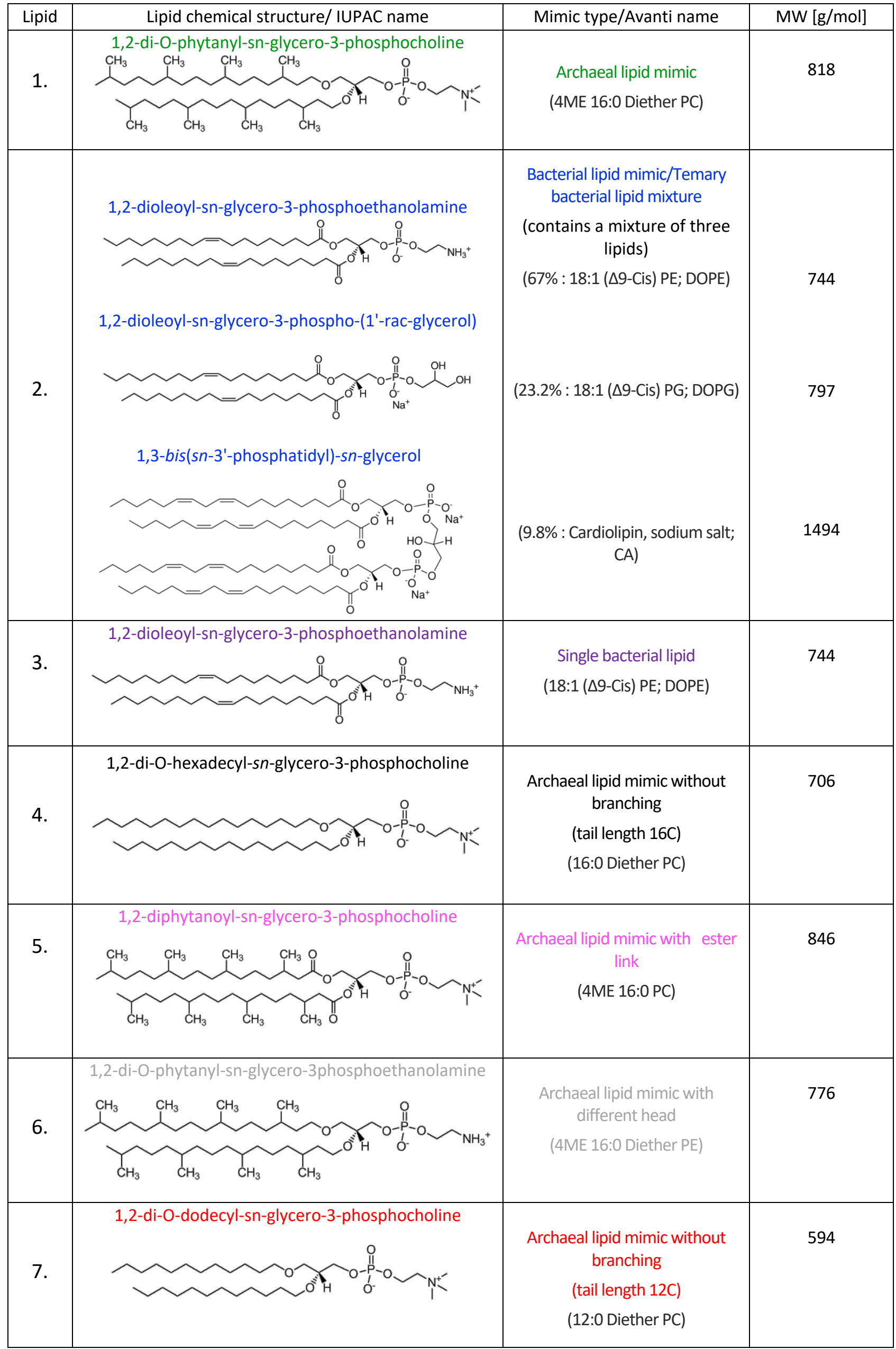


bioRxiv preprint doi: https://doi.org/10.1101/2021.10.12.464042; this version posted October 13,2021 . The copyright holder for this

preprint (which was not certified by peer review) is the author/funder, who has granted bioRxiv a license to display the preprint in perpetuity. It is made available under aCC-BY 4.0 International license.

8.

Table S1. Synthetic lipids employed to mimic archaeal and bacterial lipid membranes. List of lipids employed in this work, their chemical structure, their names according to the supplier (Avanti Polar Lipids within Merck) and their molecular weight. We could not produce vesicles using the lipids reported in grey due to technical limitations (see Methods).

\begin{tabular}{|c|c|c|c|c|c|c|c|}
\hline Metabolite & $\begin{array}{l}\text { Compound } \\
\text { Class }\end{array}$ & $\begin{array}{l}\mathrm{MW} \\
{[\mathrm{g} / \mathrm{mol}]}\end{array}$ & XLogP3 & Charge & $\begin{array}{l}\text { Rotatable } \\
\text { bond } \\
\text { counts }\end{array}$ & $\begin{array}{l}\text { Fluorescence } \\
\text { at } \mathrm{t}=3 \mathrm{~min} \\
\text { Archaea }\end{array}$ & $\begin{array}{l}\text { Fluorescence } \\
\text { at } \mathrm{t}=3 \mathrm{~min} \\
\text { Bacteria }\end{array}$ \\
\hline Glycine & Amino acid & 75 & -3.2 & 0 & 1 & 19 & 1 \\
\hline Alanine & Amino acid & 89 & -3.0 & 0 & 1 & 10 & -5 \\
\hline Leucine & Amino acid & 131 & -1.5 & 0 & 3 & 13 & 4 \\
\hline Aspartic Acid & Amino acid & 133 & -2.8 & -1 & 3 & 12 & -6 \\
\hline Lysine & Amino acid & 146 & -3.0 & +1 & 5 & 5 & 5 \\
\hline Glutamine & Amino acid & 146 & -3.1 & 0 & 4 & 24 & 2 \\
\hline Tryptophan & Amino acid & 204 & -1.1 & 0 & 3 & 0 & 2 \\
\hline Glyceraldehyde & Sugar & 90 & -1.6 & 0 & 2 & 2 & 1 \\
\hline Dihydroxyacetone & Sugar & 90 & -1.4 & 0 & 2 & 13 & 7 \\
\hline Deoxyribose & Sugar & 134 & -2.3 & 0 & 4 & 29 & 2 \\
\hline Ribose & Sugar & 150 & -2.5 & 0 & 0 & 33 & 7 \\
\hline Arabinose & Sugar & 150 & -2.5 & 0 & 0 & 9 & 6 \\
\hline Glycerol & Sugar alcohol & 92 & -1.8 & 0 & 2 & 10 & 5 \\
\hline Cytosine & Nucleobase & 111 & -1.7 & 0 & 0 & 6 & 6 \\
\hline Uracil & Nucleobase & 112 & -1.1 & 0 & 0 & 5 & 0 \\
\hline
\end{tabular}




\begin{tabular}{|l|l|l|l|l|l|l|l|}
\hline Adenine & Nucleobase & 135 & -0.1 & 0 & 0 & 16 & 5 \\
\hline Guanine & Nucleobase & 151 & -1.1 & 0 & 0 & 16 & 3 \\
\hline Urea & Amide & 60 & -1.4 & 0 & 0 & 8 & -3 \\
\hline $\begin{array}{l}\text { (2-Aminoethyl) } \\
\text { phosphonic acid }\end{array}$ & Phosphonate & 125 & -4.8 & 0 & 2 & 10 & 5 \\
\hline $\begin{array}{l}\text { Adenosine } \\
\text { monophosphate }\end{array}$ & Nucleotide & 347 & -3.5 & 0 & 4 & NA & NA \\
\hline
\end{tabular}

Table S2. Physico-chemical and permeability properties of all metabolites investigated. List of metabolites investigated in this work, the class they belong to, their molecular weight (MW), hydrophobicity (decreasing with XLogP3), their charge, the number of their rotatable bonds, the measured average fluorescence of the archaeal or bacterial lipid membrane mimic after 3 minutes exposure to each metabolite.

\begin{tabular}{|l|l|l|l|l|l|l|l|}
\hline Lipid & $\begin{array}{l}\text { Frequency } \\
{[\mathrm{Hz}]}\end{array}$ & $\begin{array}{l}\text { Amplitude } \\
{[\mathrm{V}]}\end{array}$ & $\begin{array}{l}\text { Temperature } \\
{\left[{ }^{\circ} \mathrm{C}\right]}\end{array}$ & $\begin{array}{l}\text { Time-rise } \\
{[\mathrm{min}]}\end{array}$ & $\begin{array}{l}\text { Time-main } \\
{[\mathrm{min}]}\end{array}$ & $\begin{array}{l}\text { Time-fall } \\
{[\mathrm{min}]}\end{array}$ & Outcome \\
\hline 1. & 5 & 3 & 37 & 5 & 120 & 5 & $\checkmark$ \\
\hline 2. & 10 & 1.6 & 37 & 5 & 160 & 5 & $\checkmark$ \\
\hline 3. & 5 & 3 & 37 & 5 & 120 & 5 & $\checkmark$ \\
\hline 4. & 5 & 3 & 65 & 5 & 120 & 5 & $\checkmark$ \\
\hline 5. & 5 & 3 & 37 & 5 & 120 & 5 & $\checkmark$ \\
\hline 6. & 5 & 3 & 37 & 5 & 120 & 5 & $X$ \\
\hline 6. & 5 & 3 & 65 & 5 & 120 & 5 & \\
\hline 6. & 500 & 5.3 & 65 & 5 & 120 & 5 & \\
\hline 7. & 500 & 5.3 & 65 & 5 & 120 & 5 & \\
\hline 8. & 500 & 5.3 & 65 & 5 & 120 & 5 & $\checkmark$ \\
\hline 9. & 5 & 3 & 65 & 5 & 120 & 5 & $\checkmark$ \\
\hline 9. & 5 & 3 & 65 & 5 & 120 & 5 & $X$ \\
\hline 10. & 5 & 3 & 65 & 5 & 120 & 5 & $X$ \\
\hline 11. & 500 & 5.3 & & 120 & 5 & $X$ \\
\hline
\end{tabular}

Table S3. Protocols to obtain vesicles using synthetic lipids. List of protocols employed in this work. The protocols reported in grey yielded a negative outcome (see Methods).

Supplementary file 1. Identification of putative archaeal transporter families. Family: protein family identifier. Sequences: the number of archaeal proteins within a given protein family. Crossover: the percentage of proteins within a given family that were identified by TCDB HMM searches. TM domains: the median number of transmembrane domains per protein across sequences within a protein family. Archaeal_example_seq: a single UniProt accession number of an archaeal protein assigned to a given protein family. Archaeal_taxa: the number of archaeal species encoding proteins assigned to a given family. Bacterial_taxa: the number of bacterial species that contain homologs of a given archaeal protein family based on BLAST comparisons. Putative_transporter: a classification of whether or not a given protein family likely constitutes a transporter based on TCDB overlap and the presence of transporter-associated PFAM domains. Transporter_PFAMs: all PFAM domains assigned to a protein family that were assigned a transporter-associated domain. TopAnnotation: the majority-rule annotation for a given protein family assigned using eggNOG mapper. \%withAnnot: the percentage of sequences that were assigned the majority-rule annotation. TotalAnnotSeqs: 
the number of sequences within the protein family that were successfully annotated. TotalSeqs: the total number of sequences analysed.

Video S1. After a vesicle labelled with Texas Red is confined in a microfluidic cove, the amount of fluid entering to the cove is reduced thus decreasing the probability that a second vesicle enters the cove while perimitting molecular diffusion in the cove. 